\title{
Asymptotic Capacity Analysis for Adaptive Transmission Schemes under General Fading Distributions
}

\author{
Yuan Zhang, Cihan Tepedelenlioğlu, Member, IEEE
}

\begin{abstract}
Asymptotic comparisons of ergodic channel capacity at high and low signal-to-noise ratios (SNRs) are provided for several adaptive transmission schemes over fading channels with general distributions, including optimal power and rate adaptation, rate adaptation only, channel inversion and its variants. Analysis of the high-SNR pre-log constants of the ergodic capacity reveals the existence of constant capacity difference gaps among the schemes with a pre-log constant of 1 . Closed-form expressions for these high-SNR capacity difference gaps are derived, which are proportional to the SNR loss between these schemes in $\mathrm{dB}$ scale. The largest one of these gaps is found to be between the optimal power and rate adaptation scheme and the channel inversion scheme. Based on these expressions it is shown that the presence of space diversity or multi-user diversity makes channel inversion arbitrarily close to achieving optimal capacity at high SNR with sufficiently large number of antennas or users. A low-SNR analysis also reveals that the presence of fading provably always improves capacity at sufficiently low SNR, compared to the additive white Gaussian noise (AWGN) case. Numerical results are shown to corroborate our analytical results.
\end{abstract}

\section{Index Terms}

Capacity, Power Adaptation, Fading Channels, Diversity

\section{INTRODUCTION}

In order to perform spectrally efficient communications over fading channels, adaptive transmission schemes are usually employed, which use variable transmission power or rate according

The authors are with the School of Electrical, Computer, and Energy Engineering, Arizona State University, Tempe, AZ 85287, USA. (Email: yzhang93@asu.edu, cihan@asu.edu). 
to the instantaneous channel condition, possibly along with channel coding. Determining the capacity of an adaptive transmission scheme under a given fading channel distribution is of fundamental importance. Specifically, ergodic capacity is usually used as a performance benchmark for schemes over stationary ergodic channels. In [1] the authors derive the optimal capacity with channel state information (CSI) available at both the transmitter and the receiver, which yields water-filling in time, and compare it with two sub-optimal channel inversion (CI) and truncated channel inversion (TCI) schemes. In [2] and [3] ergodic capacity is derived for fading channels modeled with generalized-k distribution, and G-distribution respectively. Both fading models are parametric and describe practical composite multi-path fading together with shadowing. Reference [4] addresses ergodic capacity with multi-user diversity, while [5], [6] analyze capacity under different diversity combining schemes. Capacity under diversity combining in the presence of spatial correlation and channel estimation error are analyzed in [7]-[10] and [11] respectively. For implementation issues, [12] presents a class of adaptive uncoded $M$-ary quadrature amplitude modulation (MQAM) schemes, and [13] presents a class of adaptive turbo-coded modulation schemes.

To the best of our knowledge, analytical comparisons of ergodic channel capacity among adaptive transmission schemes for general non-parametric channel distributions at high and low SNRs have not yet been explored. In this paper, we provide asymptotic capacity analysis at high and low SNRs for several schemes. We consider optimal, and low-complexity suboptimal adaptive transmission schemes including CI and its variants, and compare them with the corresponding AWGN capacity. By analyzing the high-SNR pre-log constants (i.e. the limiting ratio of channel capacity to the logarithm of the average SNR) of all the capacity curves, we discover the existence of constant capacity difference gaps among different schemes, and express these in closed form. Based on these results, it is shown that fading always results in worse capacity than AWGN at sufficiently high SNR regardless of the fading distribution and the scheme used. Perhaps more surprising is that arbitrary fading always improves ergodic capacity of the optimal scheme at sufficiently low SNR, compared to the equivalent AWGN channel. In addition, we confirm that the largest capacity difference gap among all the schemes is the one between the CI scheme and the optimal power and rate adaptation scheme.

Having expressions of gaps at high SNR between optimal and sub-optimal schemes for general channel distributions, we consider examples of when these sub-optimality gaps are reduced in 
the presence of diversity. Clearly, the presence of diversity improves channel capacity. What is less clear is whether the presence of diversity closes the capacity difference gap at high SNR between optimal and sub-optimal schemes. We provide the example of space diversity in which it is proved that CI comes arbitrarily close to the optimal capacity at high SNR, with sufficiently large number of transmit/receive antennas. Another example proves the same with sufficiently large number of users for the case of multi-user diversity. The rate with which the sub-optimality gap reduces with the number of antennas or users is also quantified, and it is shown that antennas are more efficient than users in reducing this gap. We also include comparisons among selected schemes which apply to the low SNR region, showing analytically that AWGN capacity is always exceeded by the optimal power and rate adaptation scheme under fading at sufficiently low SNR, which is opposite to the high SNR case already mentioned. Another interesting result establishes that the presence of outage deteriorates the high-SNR capacity, exhibiting a pre-log constant less than 1. Ultimately these results help to identify the trade-off between capacity and complexity, since CI and TCI are known to require less complex coding schemes than optimal power and rate adaptation [1] to achieve the ergodic capacity. Our main novelties, therefore, are in the consideration of general fading distributions at high and low SNR regimes, and in identifying the presence of diversity as a mechanism by which low-complexity CI schemes are near-optimal.

The rest of the paper is organized as follows. Section II establishes the channel and system model. Section III reviews the capacities of different adaptive transmission schemes. Section IV provides asymptotic analysis of these schemes, for both high and low SNR regions. Section V analyzes how the high-SNR capacity difference gaps behave under certain situations of diversity, and reveals possible near-optimality of CI in the presence of diversity. Simulation results are shown in Section VI, and Section VII concludes the paper.

\section{Channel And System Model}

Consider the following model:

$$
y=\sqrt{\rho} s+\nu
$$

where $\rho$ is the instantaneous channel power gain satisfying $\mathrm{E}[\rho]=\bar{\rho}<\infty, s$ is the transmitted sample, $y$ is the received sample, and $\nu$ is AWGN with variance $N_{0}$. Note that (1) represents an equivalent fading channel model capturing many situations other than single-input single-output 
(SISO) systems with equivalent SISO characterizations, such as multi-input single-output (MISO) and single-input multi-output (SIMO) systems. Assume the average transmission power is fixed to be $\mathrm{E}\left[|s|^{2}\right]=S$. In this case, the received instantaneous SNR becomes $\rho|s|^{2} / \mathrm{E}\left[|\nu|^{2}\right]=D \gamma$, where $D \triangleq|s|^{2} / S$ denotes the instantaneous power ratio, and $\gamma=S \rho / N_{0}$ denotes the instantaneous SNR with constant power $S$. We assume $\gamma$ has probability density function (PDF) $f_{\gamma}(x)$ and cumulative distribution function $(\mathrm{CDF}) F_{\gamma}(x)$. Notice that for adaptive transmission schemes, $D$ may become a function of $\gamma$ since the instantaneous power can be adapted to the channel. The ergodic channel capacity is given by

$$
C=\int_{0}^{\infty} \log (1+D(x) x) f_{\gamma}(x) d x=\mathrm{E}[\log (1+D(\gamma) \gamma)]
$$

per unit bandwidth, where the logarithm is natural so that $C$ is in nats per channel use. Note that $C$ depends on the average transmit power $S$ through $f_{\gamma}(x)$.

The distribution of the instantaneous SNR random variable $\gamma$ is not assumed to be of any specific parametric form for our high and low SNR results. We do however have some regularity conditions and make one or both of the following assumptions on $\gamma$ for the different results in the sequel:

- A1: $F_{\gamma}(x)$ is regularly varying at 0 [14, VIII.8]: $F_{\gamma}(x)=x^{d} l(x)$, where $0<d<\infty$ and $l(x)$ is a slowly varying function at 0 , which by definition satisfies $\lim _{x \rightarrow 0} l(\tau x) / l(x)=1$ for $\tau>0$;

- A2: $0<\mathrm{E}\left[\gamma^{-1}\right]<\infty$ (when A1 holds, this occurs if $d>1$ ).

Assumption A1 implies that $F_{\gamma}(x)$ behaves like $x^{d}$ near $x=0$, which can be shown to yield a diversity order of $d$ (for a similar result please see [15]). It also implies that $F_{\gamma}(0)=0$ and $|\mathrm{E}[\log \gamma]|<\infty$ which are proved in Appendix I. Note that $F_{\gamma}(0)=0$ requires that the fading distribution has no point of mass at the origin which means that the PDF cannot have impulses at the origin. A1 is satisfied by all channel distributions considered in the literature and will be used in the sequel. Assumption A2 ensures that the capacity of CI is nonzero and holds in the presence of space or multi-user diversity. In the sequel, A1 is necessary for most results, while A2 is necessary for results pertaining to CI and TCI and not needed for other results.

\section{AdAPTIVE TRANSMISSION SCHEMES}

In this section, we briefly review different adaptive transmission schemes, since their expressions will be needed to derive our asymptotic results. We begin with the capacity of the 
AWGN channel with the same SNR as the average SNR of the fading channel in (1) which is $\mathrm{E}[\gamma]=S \bar{\rho} / N_{0}$,

$$
C_{\mathrm{AWGN}}=\log \left(1+\frac{S \bar{\rho}}{N_{0}}\right) .
$$

In what follows, we describe several schemes and formulate their ergodic capacities, based on which we will perform asymptotic analysis at high and low SNRs.

\section{A. Optimal Power and Rate Adaptation (OA)}

We first consider the optimal power and rate adaptation scheme subject to normalized average power constraint $\mathrm{E}[D(\gamma)]=1$. As per [1], the optimized $D(\gamma)$ is given by

$$
D(\gamma)=\left(\frac{1}{\gamma_{\mathrm{t}}}-\frac{1}{\gamma}\right) I\left[\gamma>\gamma_{\mathrm{t}}\right]
$$

where $\gamma_{\mathrm{t}}$ is a threshold determined by the average power constraint

$$
\int_{\gamma_{\mathrm{t}}}^{\infty}\left(\frac{1}{\gamma_{\mathrm{t}}}-\frac{1}{x}\right) f_{\gamma}(x) d x=1
$$

and $I[\cdot]$ is the indicator function. Clearly $\gamma_{\mathrm{t}}$ is uniquely determined, since the left hand side of (5) is monotonically decreasing with $\gamma_{t}$. The optimal capacity becomes [1]

$$
C_{\mathrm{OA}}=\int_{\gamma_{\mathrm{t}}}^{\infty} \log \left(\frac{x}{\gamma_{\mathrm{t}}}\right) f_{\gamma}(x) d x .
$$

\section{B. Rate Adaptation with Receive CSI only (RA)}

We now consider another scheme in which the instantaneous power is independent of the channel (i.e. $D(\gamma)=1$ for any channel realization). The capacity is simply given as

$$
C_{\mathrm{RA}}=\int_{0}^{\infty} \log (1+x) f_{\gamma}(x) d x .
$$

As pointed out in [1], (7) is applicable as a benchmark capacity for schemes with receiver side CSI only, provided that the input distribution which maximizes mutual information is the same regardless of the channel state [16]. This holds for fading channels with AWGN [1]. Note that $C_{\mathrm{RA}} \leq C_{\mathrm{AWGN}}$ due to Jensen's inequality, while no such relation can be easily established between $C_{\mathrm{OA}}$ and $C_{\mathrm{AWGN}}$ for all $S$. 


\section{Channel Inversion (CI) with Variable Power and Constant Rate}

As its name indicates, $\mathrm{CI}$ is a scheme under which the transmission power is adapted according to the channel so that the instantaneous received SNR is kept constant. If $\mathbf{A 2}$ holds so that $\mathrm{E}\left[\gamma^{-1}\right]<\infty$, we define $D(\gamma)=\left(\mathrm{E}\left[\gamma^{-1}\right] \gamma\right)^{-1}$ so that $\mathrm{E}[D(\gamma)]=1$, which yields the constant instantaneous received $\mathrm{SNR}, \mathrm{E}^{-1}\left[\gamma^{-1}\right]$, with the corresponding capacity

$$
C_{\mathrm{CI}}=\log \left(1+\mathrm{E}^{-1}\left[\gamma^{-1}\right]\right) .
$$

Since CI effectively turns a fading channel into an AWGN channel, any coding scheme that is suitable over AWGN channels would be appropriate for CI, which is not the case for OA or RA. Therefore $\mathrm{CI}$ is considered to be a viable sub-optimal scheme due to its low complexity [1].

\section{Truncated Channel Inversion (TCI)}

For the $\mathrm{CI}$ scheme we have described, $\mathbf{A 2}$ is needed for it to have non-zero capacity. We now consider a variant of $\mathrm{CI}$ which is applicable without $\mathbf{A 2}$ and has instantaneous power ratio given by

$$
D(\gamma)=\left\{\begin{array}{ll}
0 & \text { if } \gamma<\gamma_{\mathrm{t}} \\
D_{\max } \gamma_{\mathrm{t}} / \gamma & \text { if } \gamma \geq \gamma_{\mathrm{t}}
\end{array} .\right.
$$

In (9) the transmission is ceased when $\gamma$ is below the threshold $\gamma_{\mathrm{t}}$, and $D_{\max }$ is related to the threshold $\gamma_{\mathrm{t}}$ through the average power constraint

$$
\int_{\gamma_{\mathrm{t}}}^{\infty} \frac{D_{\max } \gamma_{\mathrm{t}}}{x} f_{\gamma}(x) d x=1
$$

From (10), we obtain $D_{\max }$ as a function of $\gamma_{t}$,

$$
D_{\max }\left(\gamma_{\mathrm{t}}\right)=\left[\int_{\gamma_{\mathrm{t}}}^{\infty} \frac{\gamma_{\mathrm{t}}}{x} f_{\gamma}(x) d x\right]^{-1}
$$

and the capacity is given by

$$
C_{\mathrm{TCI}}=\left[1-F_{\gamma}\left(\gamma_{t}\right)\right] \log \left(1+D_{\max } \gamma_{\mathrm{t}}\right) .
$$




\section{E. Continuous-power Truncated Channel Inversion (CTCI)}

We now consider another variant of CI which, like TCI, is applicable without A2:

$$
D(\gamma)=\left\{\begin{array}{ll}
D_{\max } & \text { if } \gamma<\gamma_{\mathrm{t}} \\
D_{\max } \gamma_{\mathrm{t}} / \gamma & \text { if } \gamma \geq \gamma_{\mathrm{t}}
\end{array} .\right.
$$

Unlike (9), (13) is a continuous function of $\gamma$ and does not exhibit outage. In Section IV the effect of outage on capacity at both high and low SNRs will be clearly delineated. CTCI generalizes RA $\left(\gamma_{t}=\infty\right)$ and CI $\left(\gamma_{t}=0\right)$, and will be useful in our subsequent analysis to compare these schemes. Similar to TCI, $D_{\max }$ is related to the threshold $\gamma_{\mathrm{t}}$ through the average power constraint

$$
D_{\max } F_{\gamma}\left(\gamma_{\mathrm{t}}\right)+\int_{\gamma_{\mathrm{t}}}^{\infty} \frac{D_{\max } \gamma_{\mathrm{t}}}{x} f_{\gamma}(x) d x=1 .
$$

From (14), we obtain $D_{\max }$ as a function of $\gamma_{\mathrm{t}}$ given by

$$
D_{\max }\left(\gamma_{\mathrm{t}}\right)=\left[F_{\gamma}\left(\gamma_{\mathrm{t}}\right)+\int_{\gamma_{\mathrm{t}}}^{\infty} \frac{\gamma_{\mathrm{t}}}{x} f_{\gamma}(x) d x\right]^{-1} .
$$

Consequently, the capacity is given by

$$
C_{\mathrm{CTCI}}=\int_{0}^{\gamma_{\mathrm{t}}} \log \left(1+D_{\max } x\right) f_{\gamma}(x) d x+\left[1-F_{\gamma}\left(\gamma_{\mathrm{t}}\right)\right] \log \left(1+D_{\max } \gamma_{\mathrm{t}}\right) .
$$

\section{ASYMPTOTIC COMPARISONS}

We now compare the ergodic capacities of different schemes based on their asymptotic properties at both high and low average SNRs. We start by analyzing their high-SNR prelog constants, and then determine the constant capacity differences among those schemes that have a high-SNR pre-log constant of 1 . We also provide comparisons of selected schemes for low average SNR. In order to perform the subsequent asymptotic analysis, we need to separate the channel-dependent and channel-independent parameters which are involved in the capacity formulae. Since $\gamma$ is a linear function of $S$, we define the effective channel gain $z_{\text {eff }}:=\gamma / S$ whose PDF $f_{z_{\text {eff }}}(z)$ and $\operatorname{CDF} F_{z_{\text {eff }}}(z)$ are only related to the channel distribution, so that $z_{\text {eff }}$ does not depend on the average power $S$. Similarly, we define the threshold $z_{\mathrm{t}}:=\gamma_{\mathrm{t}} / S$ to facilitate our analysis. 


\section{A. Asymptotic Slopes and Pre-log Constants of Capacity Curves}

We now consider each of the schemes in Section III to determine how their capacities behave at both high and low SNRs. For any capacity expression of the form $C=\mathrm{E}\left[\log \left(1+S D\left(z_{\mathrm{eff}}\right) z_{\mathrm{eff}}\right)\right]$ we analyze the pre-log constant $\lim _{S \rightarrow \infty} d C / d(\log S)$ as defined e.g. in [17]. We also examine the low-SNR slope $\lim _{S \rightarrow 0} d C / d S$ since in most cases $C$ becomes approximately linear with $S$ as $S \rightarrow 0$. For the AWGN capacity given by (3), we have the well-known

$$
\begin{aligned}
& \lim _{S \rightarrow 0} \frac{d C_{\mathrm{AWGN}}}{d S}=\frac{\bar{\rho}}{N_{0}}=\mathrm{E}\left[z_{\mathrm{eff}}\right] \\
& \lim _{S \rightarrow \infty} \frac{d C_{\mathrm{AWGN}}}{d(\log S)}=1
\end{aligned}
$$

as a benchmark, where $\mathrm{E}\left[z_{\text {eff }}\right]$ is finite in (17) since we have assumed $\bar{\rho}<\infty$. We proceed with the fading case starting with the optimal power and rate adaptation.

1) Optimal Power and Rate Adaptation: For the case of OA, we rewrite (5) and (6) as

$$
\begin{aligned}
& \int_{z_{\mathrm{t}}}^{\infty} \frac{1}{S}\left(\frac{1}{z_{\mathrm{t}}}-\frac{1}{z}\right) f_{z_{\mathrm{eff}}}(z) d z=1 \\
& C_{\mathrm{OA}}=\int_{z_{\mathrm{t}}}^{\infty} \log \left(\frac{z}{z_{\mathrm{t}}}\right) f_{z_{\mathrm{eff}}}(z) d z .
\end{aligned}
$$

We express the slope and pre-log constant in terms of $z_{\mathrm{t}}$ since $C_{\mathrm{OA}}$ is implicitly related to $S$ through $z_{\mathrm{t}}$ in (19). Differentiating (20) with respect to $z_{\mathrm{t}}$, we obtain

$$
\frac{d C_{\mathrm{OA}}}{d z_{\mathrm{t}}}=\frac{F_{z_{\mathrm{eff}}}\left(z_{\mathrm{t}}\right)-1}{z_{\mathrm{t}}}
$$

Solving for $S$ from (19) as a function of $z_{\mathrm{t}}$, we obtain

$$
S z_{\mathrm{t}}=1-F_{z_{\mathrm{eff}}}\left(z_{\mathrm{t}}\right)-z_{\mathrm{t}} \int_{z_{\mathrm{t}}}^{\infty} \frac{1}{z} f_{z_{\mathrm{eff}}}(z) d z
$$

and

$$
\frac{d z_{\mathrm{t}}}{d S}=\frac{z_{\mathrm{t}}^{2}}{F_{z_{\mathrm{eff}}}\left(z_{\mathrm{t}}\right)-1}
$$

Consequently we have

$$
\frac{d C_{\mathrm{OA}}}{d S}=\frac{d C_{\mathrm{OA}}}{d z_{\mathrm{t}}} \frac{d z_{\mathrm{t}}}{d S}=z_{\mathrm{t}}
$$

and

$$
\frac{d C_{\mathrm{OA}}}{d(\log S)}=S \frac{d C_{\mathrm{OA}}}{d S}=S z_{\mathrm{t}}=1-F_{z_{\mathrm{eff}}}\left(z_{\mathrm{t}}\right)-z_{\mathrm{t}} \int_{z_{\mathrm{t}}}^{\infty} \frac{1}{z} f_{z_{\mathrm{eff}}}(z) d z .
$$

To determine the limits of (24) and (25), we have the following lemma. 
Lemma 1: The function $S \mapsto z_{\mathrm{t}}$ defined implicitly through (19) has $\operatorname{limits} \lim _{S \rightarrow 0} z_{\mathrm{t}}=$ $\sup \left\{z: F_{z_{\mathrm{eff}}}(z)<1\right\}$ and $\lim _{S \rightarrow \infty} z_{\mathrm{t}}=0$.

Proof: See Appendix II.

Note that for commonly considered fading channels, including Nakagami- $m$, Ricean and lognormal, $z_{\text {eff }}$ has an infinite support and thus $\sup \left\{z: F_{z_{\mathrm{eff}}}(z)<1\right\}=\infty$. Using Lemma 1 and (25) we have

$$
\lim _{S \rightarrow \infty} \frac{d C_{\mathrm{OA}}}{d(\log S)}=\lim _{z_{\mathrm{t}} \rightarrow 0^{+}}\left(1-F_{z_{\mathrm{eff}}}\left(z_{\mathrm{t}}\right)-z_{\mathrm{t}} \int_{z_{\mathrm{t}}}^{\infty} \frac{1}{z} f_{z_{\mathrm{eff}}}(z) d z\right)
$$

Clearly $\lim _{z_{\mathrm{t}} \rightarrow 0^{+}} z_{\mathrm{t}} \int_{z_{\mathrm{t}}}^{\infty} z^{-1} f_{z_{\mathrm{eff}}}(z) d z=0$ when $\mathbf{A} 2$ is satisfied; otherwise, based on L'Hôpital's rule $\lim _{z_{\mathrm{t}} \rightarrow 0^{+}} z_{\mathrm{t}} \int_{z_{\mathrm{t}}}^{\infty} z^{-1} f_{z_{\mathrm{eff}}}(z) d z=\lim _{z_{\mathrm{t}} \rightarrow 0^{+}} z_{\mathrm{t}} f_{z_{\mathrm{eff}}}\left(z_{\mathrm{t}}\right)=\lim _{z_{\mathrm{t}} \rightarrow 0^{+}} F_{z_{\mathrm{eff}}}\left(z_{\mathrm{t}}\right)-F_{z_{\mathrm{eff}}}(0)=0$. Consequently, $\lim _{S \rightarrow \infty} d C_{\mathrm{OA}} / d(\log S)=1-F_{z_{\mathrm{eff}}}(0)$. Knowing that $\mathbf{A 1}$ holds and $z_{\mathrm{eff}}=\gamma / S$ we have $F_{z_{\text {eff }}}(0)=0$. The asymptotic slope and pre-log constant are given by

$$
\begin{aligned}
\lim _{S \rightarrow 0} \frac{d C_{\mathrm{OA}}}{d S} & =\sup \left\{z: F_{z_{\mathrm{eff}}}(z)<1\right\} \\
\lim _{S \rightarrow \infty} \frac{d C_{\mathrm{OA}}}{d(\log S)} & =1
\end{aligned}
$$

by taking the limits of (24) and (25). As we will see from subsequent derivations, (27) and (28) are the largest possible for any scheme in the presence of fading, which is expected since OA is the optimal scheme for all $S$. Typically when $z_{\text {eff }}$ has infinite support, $\lim _{S \rightarrow 0} d C_{\mathrm{OA}} / d S=$ $\sup \left\{z: F_{z_{\mathrm{eff}}}(z)<1\right\}=\infty$ and thus OA becomes better than AWGN at low average SNR given (17). We will include further discussions of this issue in Subsection IV-D.

2) Truncated Channel Inversion: For TCI, we rewrite (11) and (12) as

$$
\begin{aligned}
D_{\max }\left(z_{\mathrm{t}}\right) & =\left[\int_{z_{\mathrm{t}}}^{\infty} \frac{z_{\mathrm{t}}}{z} f_{z_{\mathrm{eff}}}(z) d z\right]^{-1} \\
C_{\mathrm{TCI}} & =\left[1-F_{z_{\mathrm{eff}}}\left(z_{\mathrm{t}}\right)\right] \log \left(1+S D_{\max }\left(z_{\mathrm{t}}\right) z_{\mathrm{t}}\right) .
\end{aligned}
$$

Equation (29) indicates that $D_{\max }$ does not depend on $S$ given $z_{\mathrm{t}}$. By differentiating (30) with respect to $S$, we obtain

$$
\frac{d C_{\mathrm{TCI}}}{d S}=\frac{D_{\max } z_{\mathrm{t}}}{1+S D_{\max } z_{\mathrm{t}}}\left[1-F_{z_{\mathrm{eff}}}\left(z_{\mathrm{t}}\right)\right]
$$

Setting $S=0$ and substituting (29) in (31), we obtain the asymptotic slope at low average SNR

$$
\lim _{S \rightarrow 0} \frac{d C_{\mathrm{TCI}}}{d S}=z_{\mathrm{t}} D_{\max }\left[1-F_{z_{\mathrm{eff}}}\left(z_{\mathrm{t}}\right)\right]=\frac{1-F_{z_{\mathrm{eff}}}\left(z_{\mathrm{t}}\right)}{\int_{z_{\mathrm{t}}}^{\infty} \frac{1}{z} f_{z_{\mathrm{eff}}}(z) d z}
$$


which can be verified to be monotonically increasing with $z_{\mathrm{t}}$ in the support of $f_{z_{\mathrm{eff}}}(z)$ and has a maximum value of $\sup \left\{z: F_{z_{\text {eff }}}(z)<1\right\}$. At high average SNR we have

$$
\lim _{S \rightarrow \infty} \frac{d C_{\mathrm{TCI}}}{d(\log S)}=\lim _{S \rightarrow \infty} \frac{S d C_{\mathrm{TCI}}}{d S}=1-F_{z_{\mathrm{eff}}}\left(z_{\mathrm{t}}\right)
$$

which is monotonically decreasing with $z_{\mathrm{t}}$ in the support of $f_{z_{\mathrm{eff}}}(z)$.

Recall that (32) and (33) are derived assuming $z_{\mathrm{t}}$ to be a fixed threshold, independent of $S$. This is unlike our discussion of the OA scheme where $z_{\mathrm{t}}$ is optimized for each $S$. Such an optimization can also be adopted in this TCI context by maximizing (30) with respect to $z_{\mathrm{t}}$. To distinguish it from a fixed threshold we will denote the maximizer of (30) by $z_{\mathrm{t}}^{*}(S)$. Even though it is not possible to express $z_{\mathrm{t}}^{*}(S)$ in closed form, one can still analyze asymptotic expressions for a TCI scheme with optimal threshold. In the case of the pre-log constant, it is clear that the optimized TCI should outperform any TCI scheme with fixed $z_{\mathrm{t}}$. From (33) we see that the pre-log constant of the optimized TCI should be at least $\sup _{z_{\mathrm{t}}}\left(1-F_{z_{\text {eff }}}\left(z_{\mathrm{t}}\right)\right)=1$ when A1 holds which is the best achievable pre-log constant over fading channels in (28). Similarly, $z_{\mathrm{t}}^{*}(S)$ should approach $\sup \left\{z: F_{z_{\mathrm{eff}}}(z)<1\right\}$ as $S \rightarrow 0$ since (32) is monotonically increasing with $z_{\mathrm{t}}$.

3) Continuous-power Truncated Channel Inversion: We next consider the case of CTCI which unifies RA (with $z_{\mathrm{t}}=\infty$ ) and CI (with $z_{\mathrm{t}}=0$ ). Equations (15) and (16) can be rewritten as

$$
\begin{aligned}
D_{\max }\left(z_{\mathrm{t}}\right) & =\left[F_{z_{\mathrm{eff}}}\left(z_{\mathrm{t}}\right)+\int_{z_{\mathrm{t}}}^{\infty} \frac{z_{\mathrm{t}}}{z} f_{z_{\mathrm{eff}}}(z) d z\right]^{-1} \\
C_{\mathrm{CTCI}} & =\int_{0}^{z_{\mathrm{t}}} \log \left(1+S D_{\max } z\right) f_{z_{\mathrm{eff}}}(z) d z+\left[1-F_{z_{\mathrm{eff}}}\left(z_{\mathrm{t}}\right)\right] \log \left(1+S D_{\mathrm{max}} z_{\mathrm{t}}\right) .
\end{aligned}
$$

Equation (34) indicates that $D_{\max }$ does not depend on $S$ given $z_{\mathrm{t}}$. By differentiating (35) with respect to $S$, we obtain

$$
\frac{d C_{\mathrm{CTCI}}}{d S}=\int_{0}^{z_{\mathrm{t}}} \frac{D_{\max } z}{1+S D_{\max } z} f_{z_{\mathrm{eff}}}(z) d z+\frac{D_{\max } z_{\mathrm{t}}}{1+S D_{\max } z_{\mathrm{t}}}\left[1-F_{z_{\mathrm{eff}}}\left(z_{\mathrm{t}}\right)\right] .
$$

Setting $S=0$ and substituting (34) in (36), we obtain the asymptotic slope at low average SNR $\lim _{S \rightarrow 0} d C_{\mathrm{CTCI}} / d S=\left(\int_{0}^{z_{\mathrm{t}}} z f_{z_{\mathrm{eff}}}(z) d z+z_{\mathrm{t}}\left[1-F_{z_{\mathrm{eff}}}\left(z_{\mathrm{t}}\right)\right]\right) /\left(F_{z_{\mathrm{eff}}}\left(z_{\mathrm{t}}\right)+\int_{z_{\mathrm{t}}}^{\infty} z_{\mathrm{t}} z^{-1} f_{z_{\mathrm{eff}}}(z) d z\right)$, where we exchange the limit and integral in the first term, since the absolute value of the integrand is upper bounded by $D_{\max } z f_{z_{\mathrm{eff}}}(z)$ which is an integrable function, and the condition for dominated convergence is satisfied. Notice that this general formula for $\lim _{S \rightarrow 0} d C_{\mathrm{CTCI}} / d S$ is suitable for 
$0<z_{\mathrm{t}}<\infty$ only, while for the cases of RA $\left(z_{\mathrm{t}}=\infty\right)$ and CI $\left(z_{\mathrm{t}}=0\right)$, we can simply rewrite (7) and (8) as

$$
\begin{aligned}
C_{\mathrm{RA}} & =\int_{0}^{\infty} \log (1+S z) f_{z_{\mathrm{eff}}}(z) d z \\
C_{\mathrm{CI}} & =\log \left(1+S \mathrm{E}^{-1}\left[z_{\mathrm{eff}}^{-1}\right]\right)
\end{aligned}
$$

and obtain

$$
\begin{aligned}
& \lim _{S \rightarrow 0} \frac{d C_{\mathrm{RA}}}{d S}=\mathrm{E}\left[z_{\mathrm{eff}}\right] \\
& \lim _{S \rightarrow 0} \frac{d C_{\mathrm{CI}}}{d S}=\mathrm{E}^{-1}\left[z_{\mathrm{eff}}^{-1}\right] .
\end{aligned}
$$

For the analysis at high average SNR, based on (36) we obtain

$$
\lim _{S \rightarrow \infty} \frac{d C_{\mathrm{CTCI}}}{d(\log S)}=\lim _{S \rightarrow \infty} \frac{S d C_{\mathrm{CTCI}}}{d S}=\int_{0}^{z_{\mathrm{t}}} f_{z_{\mathrm{eff}}}(z) d z+1-F_{z_{\mathrm{eff}}}\left(z_{\mathrm{t}}\right)=1
$$

which is independent of the threshold. Here we exchange the limit and integral since the integrand is upper bounded by an integrable function, $f_{z_{\text {eff }}}(z)$, and the condition for the dominated convergence theorem is satisfied. Notice that (41) also applies to RA and CI since they are special cases of CTCI. By comparing (33) and (41), we notice that TCI has a smaller pre-log constant than CTCI at high average SNR and thus has a worse capacity in that regime. We will also give their capacity comparison at low average SNR in Subsection IV-D, where the opposite will be seen to hold.

Similar to OA and TCI one can seek to optimize $z_{\mathrm{t}}$ in (35) for each $S$ instead of using a fixed $z_{\mathrm{t}}$. Interestingly, unlike the cases of OA and TCI, maximizing (35) over $z_{\mathrm{t}}$ yields the trivial answer of $z_{\mathrm{t}}=\infty$, as stated in the following theorem.

Theorem 1: $C_{\mathrm{CTCI}}$ as given by (35) is monotonically increasing with $z_{\mathrm{t}}$ for any value of $S$.

Proof: See Appendix III.

Since RA and CI are obtained as special cases of CTCI when $z_{\mathrm{t}}=\infty$ and $z_{\mathrm{t}}=0$ respectively, Theorem 1 indicates that $C_{\mathrm{CI}} \leq C_{\mathrm{CTCI}} \leq C_{\mathrm{RA}}$ regardless of average SNR.

\section{B. Effect of Outage on the Pre-log Constant}

Recall that TCI involves outage while CTCI does not. The pre-log constant of CTCI is 1 whereas that of TCI is strictly less than 1 . Without being restricted to the parametric forms of $D(z)$ in (9) and (13), we now generalize this result and show that the presence of outage reduces the pre-log constant for any instantaneous power ratio $D(z)$. 
Theorem 2: Let $D(z)$ be independent of $S$, define $\mathscr{O}=\{z \mid D(z)=0\}$, and assume $\mathbf{A 1}$ is satisfied. Then the pre-log constant of the scheme with instantaneous power ratio $D(z)$ is given by

$$
\lim _{S \rightarrow \infty} \frac{d \mathrm{E}\left[\log \left(1+S D\left(z_{\mathrm{eff}}\right) z_{\mathrm{eff}}\right)\right]}{d(\log S)}=1-\operatorname{Pr}(\mathscr{O})
$$

\section{Proof: See Appendix IV.}

Theorem 2 clarifies that the pre-log constant being 1 or strictly less than 1 is related to the absence or presence of outage. Applying Theorem 2, it is easy to see that the pre-log constants of RA, CI and CTCI are 1, provided that $\mathbf{A 1}$ is satisfied. In contrast, the pre-log constant of TCI is given by $1-F_{z_{\text {eff }}}\left(z_{\mathrm{t}}\right)$. Therefore, we expect constant capacity difference gaps among the capacity curves of AWGN, OA, RA, CI and CTCI at sufficiently high average SNR. In what follows, we use the term "gap" to denote $\lim _{S \rightarrow \infty}\left(C_{1}-C_{2}\right)$ for schemes 1 and 2 satisfying $\lim _{S \rightarrow \infty} d C_{1} / d(\log S)=\lim _{S \rightarrow \infty} d C_{2} / d(\log S)=1$. The corresponding gap in average SNR in $\mathrm{dB}$ can be derived to be simply $10 / \log 10$ times the capacity difference gap above.

\section{Asymptotic Gaps among AWGN, OA, RA and CI at High Average SNR}

To derive the high-SNR asymptotic capacity gaps, we introduce the following.

Lemma 2: For a constant $\mu>0$ and a non-negative random variable $z_{\text {eff }}$ having finite $\mathrm{E}\left[\log z_{\text {eff }}\right]$, we have

Proof: See Appendix V.

$$
\begin{aligned}
& \lim _{S \rightarrow \infty} \int_{0}^{\infty} \log \left(\frac{1+S \mu}{S \mu}\right) f_{z_{\mathrm{eff}}}(z) d z=0 \\
& \lim _{S \rightarrow \infty} \int_{0}^{\infty} \log \left(\frac{1+S z}{S z}\right) f_{z_{\mathrm{eff}}}(z) d z=0
\end{aligned}
$$

Notice that the assumption $-\infty<\mathrm{E}\left[\log z_{\mathrm{eff}}\right]<\infty$ we have in Lemma 2 follows from $\mathbf{A} \mathbf{1}$ and is weaker than A2, while $\mathbf{A 2}$ can also be assumed to prove Lemma 2. Consequently we have the following theorem

Theorem 3: The following high-SNR capacity difference gaps among the OA, RA, CI schemes and AWGN capacity are given by

$$
\begin{gathered}
\lim _{S \rightarrow \infty}\left(C_{\mathrm{OA}}-C_{\mathrm{RA}}\right)=0 \\
\lim _{S \rightarrow \infty}\left(C_{\mathrm{AWGN}}-C_{\mathrm{OA}}\right)=\log \left(\mathrm{E}\left[z_{\mathrm{eff}}\right]\right)-\mathrm{E}\left[\log z_{\mathrm{eff}}\right] \geq 0 \\
\lim _{S \rightarrow \infty}\left(C_{\mathrm{OA}}-C_{\mathrm{CI}}\right)=\mathrm{E}\left[\log z_{\mathrm{eff}}\right]+\log \left(\mathrm{E}\left[z_{\mathrm{eff}}^{-1}\right]\right) \geq 0
\end{gathered}
$$


Moreover, (46) is finite when A1 holds, and (47) is finite when A2 holds.

Proof: See Appendix VI.

Theorem 3 indicates that the AWGN capacity exceeds the optimal capacity under any fading channel distribution at high SNR. However, in IV-D we will show the exact opposite at low SNR. We have thus established that OA and RA exhibit zero gap at high average SNR, and that (46) yields the (non-negative) gap between AWGN and OA. We recall that as a consequence of the discussion after Theorem 1,

$$
C_{\mathrm{CI}} \leq C_{\mathrm{CTCI}} \leq C_{\mathrm{RA}} \leq C_{\mathrm{OA}}
$$

for any $S$, and therefore the gap between $C_{\mathrm{OA}}$ and $C_{\mathrm{CI}}$, given by (47), is the largest constant gap among all the schemes over fading channels we have addressed.

\section{Asymptotic Comparisons at Low Average SNR}

From the asymptotic analysis at high average SNR, we recall that the presence of outage in adaptive transmission schemes tends to result in worse capacity than no outage (Theorem 2) and AWGN capacity is better than all the capacities under fading based on (46). However, capacity comparisons at low average SNR are sharply different. We have the following results for selected schemes at low average SNR.

Theorem 4: The low-SNR slopes of capacities satisfy the following:

$$
\begin{aligned}
& \text { CTCI: } \mathrm{E}^{-1}\left[z_{\mathrm{eff}}^{-1}\right]=\lim _{S \rightarrow 0} \frac{d C_{\mathrm{CI}}}{d S} \leq \lim _{S \rightarrow 0} \frac{d C_{\mathrm{CTCI}}}{d S} \leq \lim _{S \rightarrow 0} \frac{d C_{\mathrm{RA}}}{d S}=\mathrm{E}\left[z_{\mathrm{eff}}\right] \\
& \text { OA: } \quad \lim _{S \rightarrow 0} \frac{d C_{\mathrm{OA}}}{d S}=\sup \left\{z: F_{z_{\mathrm{eff}}}(z)<1\right\} \geq \mathrm{E}\left[z_{\mathrm{eff}}\right]=\lim _{S \rightarrow 0} \frac{d C_{\mathrm{AWGN}}}{d S} \\
& \text { TCI: } \quad \mathrm{E}^{-1}\left[z_{\mathrm{eff}}^{-1}\right] \leq \lim _{S \rightarrow 0} \frac{d C_{\mathrm{TCI}}}{d S} \leq \sup \left\{z: F_{z_{\mathrm{eff}}}(z)<1\right\}
\end{aligned}
$$

where, in (49) the two inequalities become equalities when the threshold satisfies $z_{\mathrm{t}}=0$ and $\infty$ respectively; in (50) the equality holds only when the fading channel is deterministic and reduces to AWGN; in (51) the two inequalities become equalities when the threshold $z_{\mathrm{t}}=0$ and $\sup \left\{z: F_{z_{\text {eff }}}(z)<1\right\}$ respectively. Also for TCI with a threshold $z_{\mathrm{t}}, z_{\mathrm{t}} \geq \mathrm{E}\left[z_{\mathrm{eff}}\right]$ is a sufficient condition for $\lim _{S \rightarrow 0} d C_{\mathrm{TCI}} / d S \geq \lim _{S \rightarrow 0} d C_{\mathrm{AWGN}} / d S$.

Proof: See Appendix VII. 
Theorem 4 indicates that OA gives larger capacity than AWGN at low SNRs for any fading distribution, and the presence of fading always improves capacity when the average SNR is sufficiently low. To the best of our knowledge, this is proved analytically here for the first time, even though it has been briefly mentioned in [1] and addressed in [18] with a numerical example for specific distributions without an analytical proof and addressed in [19] through approximations. Also, with the choice $z_{\mathrm{t}} \geq \mathrm{E}\left[z_{\text {eff }}\right]$, TCI can also give larger capacity than AWGN at low average SNRs, which to our knowledge was never mentioned in existing literature. It follows that with a sufficiently low average SNR, the presence of fading can result in better capacity than the equivalent AWGN channel. Furthermore, the comparison between (49) and (51) suggests that outage can be helpful to improve the low-SNR capacity by exploiting the aforementioned benefit of fading.

\section{Asymptotic Optimality OF CI With Diversity}

It is clear that the presence of diversity will improve the ergodic capacity of all the schemes discussed. However, the high-SNR capacity gap between OA and the sub-optimal CI also reduces in the presence of diversity rendering CI near-optimal in some cases. In this section, we give examples of space diversity and multi-user diversity to show that the gap given by (47) between $\mathrm{CI}$ and OA can be made arbitrarily small with sufficiently large number of antennas or users. Since (46) is non-negative, $\lim _{S \rightarrow \infty}\left(C_{\mathrm{AWGN}}-C_{\mathrm{CI}}\right) \geq \lim _{S \rightarrow \infty}\left(C_{\mathrm{OA}}-C_{\mathrm{CI}}\right)$, it will also be convenient to investigate

$$
\lim _{S \rightarrow \infty}\left(C_{\mathrm{AWGN}}-C_{\mathrm{CI}}\right)=\lim _{S \rightarrow \infty}\left(C_{\mathrm{AWGN}}-C_{\mathrm{OA}}\right)+\lim _{S \rightarrow \infty}\left(C_{\mathrm{OA}}-C_{\mathrm{CI}}\right)=\log \left(\mathrm{E}\left[z_{\text {eff }}\right] \mathrm{E}\left[z_{\text {eff }}^{-1}\right]\right)
$$

in the presence of diversity. Clearly, showing that the gap in (52) can be made arbitrarily small in the presence of diversity would establish the same for (47).

\section{A. Example of Space Diversity}

For space diversity, we consider the case in which the system consists of a transmitter with $N \geq 2$ antennas and a receiver with a single antenna, and the instantaneous SNR is to be maximized through beamforming. We assume the components $\left\{h_{n}\right\}_{n=1}^{N}$ of the $N \times 1$ channel vector are independently and identically distributed (i.i.d.) circularly symmetric $\mathcal{C N}(0,1)$ random variables, and the noise term is also circularly symmetric $\mathcal{C N}(0,1)$. It is well-known that the 
maximized effective channel gain is $z_{\text {eff }}=\sum_{n=1}^{N}\left|h_{n}\right|^{2}$, whose PDF is given by $f_{z_{\mathrm{eff}}}(z)=$ $z^{N-1} e^{-z} / \Gamma(N)$. Here $\Gamma(x):=\int_{0}^{\infty} t^{x-1} e^{-t} d t$ is the Gamma function and for integer $N$ it becomes $\Gamma(N)=(N-1)$ !. It can easily be verified that $\mathbf{A 1}$ is satisfied with $d=N$. The gaps in (47) and (52) become

$$
\begin{aligned}
\lim _{S \rightarrow \infty}\left(C_{\mathrm{OA}}-C_{\mathrm{CI}}\right) & =\int_{0}^{\infty} \log z f_{z_{\mathrm{eff}}}(z) d z+\log \left(\int_{0}^{\infty} \frac{1}{z} f_{z_{\mathrm{eff}}}(z) d z\right) \\
& =\psi(N)-\log (N-1) \\
\lim _{S \rightarrow \infty}\left(C_{\mathrm{AWGN}}-C_{\mathrm{CI}}\right) & =\log \left(\int_{0}^{\infty} z f_{z_{\mathrm{eff}}}(z) d z\right)+\log \left(\int_{0}^{\infty} \frac{1}{z} f_{z_{\mathrm{eff}}}(z) d z\right) \\
& =\log N+\log \left(\frac{1}{N-1}\right)=\log \left(1+\frac{1}{N-1}\right)
\end{aligned}
$$

where $\psi(x)=d \log \Gamma(x) / d x$ is the digamma function. In [20] $C_{\mathrm{CI}}$ was derived for $N>1$ and termed delay-limited capacity, and is given by $\log (1+(N-1) S / N)$. The result in (54) can also be obtained using the expression for $C_{\mathrm{CI}}$ in [20]. However, the gap in (53) was never addressed therein. We observe from (53) and (54) that the gaps can be made arbitrarily small for sufficiently large number of antennas. Therefore, CI provides near-optimal capacity at high average SNR with space diversity. Moreover, for large $N$ we have

$$
\begin{gathered}
\psi(N)-\log (N-1)=\frac{1}{2(N-1)}+\mathcal{O}\left(N^{-2}\right) \\
\log \left(1+\frac{1}{N-1}\right)=\frac{1}{(N-1)}+\mathcal{O}\left(N^{-2}\right) .
\end{gathered}
$$

It can be seen that (53) is approximately half of (54) and they both behave inversely proportional to $N-1$ with $N$ being sufficiently large. We note that a similar result can be obtained for receive diversity instead of transmit beamforming.

\section{B. Example of Multi-user Diversity}

For multi-user diversity, we consider the case in which the transmitter selects the user (receiver) among $K \geq 2$ users with the best channel for transmission at any given time instant. Specifically, the effective channel gain becomes $z_{\text {eff }}=\max _{k=1,2, \ldots, K}\left\{z_{k}\right\}$ where $z_{k}$ is the individual channel gain when the $k$-th user is selected for transmission. In general, the problem is more complicated than space diversity since we are unable to get closed-form results of the 
gaps in (47) and (52) for the commonly considered wireless channels. However, as we now see it is possible to quantify how the gap given by (52) scales with the number of users $K$.

We investigate the case in which $z_{k}$ has exponential $\operatorname{CDF} F(z)=1-e^{-z}$, corresponding to a single-antenna system under Rayleigh fading with $K$ users. In this case, $z_{\text {eff }}$ has $\operatorname{CDF} F_{z_{\text {eff }}}(z)=$ $\left(1-e^{-z}\right)^{K}$ and PDF $f_{z_{\mathrm{eff}}}(z)=K e^{-z}\left(1-e^{-z}\right)^{K-1}$, with $d=K$ in A1. It is well-known that $\mathrm{E}\left[z_{\mathrm{eff}}\right]=\sum_{k=1}^{K} 1 / k$. Adapting Lemma 3.1 in [21], it can be shown that $\mathrm{E}\left[z_{\text {eff }}^{-1}\right] \sim 1 / \log K$ (here “ $"$ denotes asymptotical equality) as $K \rightarrow \infty$. Moreover since $\lim _{K \rightarrow \infty}\left(\sum_{k=1}^{K} 1 / k-\log K\right)=$ $\gamma_{\mathrm{em}}$ where $\gamma_{\mathrm{em}} \approx 0.5772$ is the Euler-Mascheroni constant, for $K \rightarrow \infty$ the high-SNR gap between $C_{\mathrm{AWGN}}$ and $C_{\mathrm{CI}}$ given by (52) becomes

$$
\log \left(\mathrm{E}\left[z_{\mathrm{eff}}\right] \mathrm{E}\left[z_{\mathrm{eff}}^{-1}\right]\right) \sim \log \left(\frac{\log K+\gamma_{\mathrm{em}}}{\log K}\right)=\log \left(1+\frac{\gamma_{\mathrm{em}}}{\log K}\right) \sim \frac{\gamma_{\mathrm{em}}}{\log K} .
$$

Due to the limitation of mathematical tools, we are unable to derive any asymptotic expression of $\lim _{S \rightarrow \infty}\left(C_{\mathrm{OA}}-C_{\mathrm{CI}}\right)$ rigorously under multi-user diversity, however we conjecture $\lim _{S \rightarrow \infty}\left(C_{\mathrm{OA}}-\right.$ $\left.C_{\mathrm{CI}}\right)=\mathrm{E}\left[\log z_{\text {eff }}\right]+\log \left(\mathrm{E}\left[z_{\text {eff }}^{-1}\right]\right) \sim \gamma_{\mathrm{em}} /(\log K(1+\log K))$ for sufficiently large $K$ based on a non-rigorous approach, which evaluates $\mathrm{E}\left[\log z_{\text {eff }}\right]$ and $\log \left(\mathrm{E}\left[z_{\text {eff }}^{-1}\right]\right)$ directly based on the extreme distributions of $\log z_{\text {eff }}$ and $z_{\text {eff }}^{-1}$ without verifying the condition of uniform integrability [22, ch.6]. Clearly (57) converges very slowly to zero as the number of users increases, and consequently so does the gap between OA and CI as per the discussion after (52). Therefore, multi-user diversity can also render CI achieve near-AWGN capacity, but the number of users needed to close the gap given by (57) is much larger than the number of antennas needed for (54).

It is interesting to note that for the multi-user diversity example, (47) and (52) do not always converge to zero as $K \rightarrow \infty$ if the SNR distribution of a single user is not exponential. For example, when $z_{k}$ is of Fréchet distribution with $\operatorname{CDF} F(z)=\exp \left(-z^{-\alpha}\right)$, it can be shown that (47) and (52) are given by

$$
\begin{gathered}
\mathrm{E}\left[\log z_{\mathrm{eff}}\right]+\log \left(\mathrm{E}\left[z_{\text {eff }}^{-1}\right]\right)=\frac{\gamma_{\mathrm{em}}}{\alpha}+\log \Gamma\left(1+\frac{1}{\alpha}\right) \\
\log \left(\mathrm{E}\left[z_{\mathrm{eff}}\right] \mathrm{E}\left[z_{\text {eff }}^{-1}\right]\right)=\log \left(\Gamma\left(1-\frac{1}{\alpha}\right) \Gamma\left(1+\frac{1}{\alpha}\right)\right)
\end{gathered}
$$

which do not depend on $K$ ! In such cases, it becomes impossible for CI to achieve the optimal or AWGN capacity by simply increasing the number of users. Fréchet distribution is known to arise from the extreme signal-to-interference ratio distribution in interference-limited MIMO wireless channels with sufficiently large number of users [23]. 


\section{Simulation Results}

In this section, we provide simulation results to corroborate our theoretical analysis. In all simulations we model the system as a multi-antenna and/or multi-user MISO system with $N$ antennas at the base station (transmitter) and a single antenna at each of the $K$ users (receivers). Only the user with the best channel condition is selected for transmission at each time instant. Specifically, we assume the channel coefficients between antenna $n$ and user $k, h_{n k}$, are i.i.d. circularly symmetric $\mathcal{C N}(0,1)$ random variables and so are the noise terms. Then the maximized effective channel gain becomes $z_{\text {eff }}=\max _{k=1,2, \ldots, K}\left\{\sum_{n=1}^{N}\left|h_{n k}\right|^{2}\right\}$, whose PDF is given by $f_{z_{\text {eff }}}(z)=K(\Gamma(N))^{-K}[\gamma(N, z)]^{K-1} z^{N-1} e^{-z}$. It can be proved that $\mathbf{A 2}$ holds if and only if $\max (N, K) \geq 2$, i.e. at least one kind of diversity is present. Recall that the average SNR is the average power $S$ in linear scale since the channel noise has normalized variance. In the plots, we exhibit our results using base-2 logarithm instead of natural logarithm, so that all the capacity values and gaps are in bits per channel use.

Figure 1 shows the capacity curves of OA, RA, CI and AWGN for $(N, K)=(2,2)$. We observe that RA achieves almost the same capacity as OA. In addition, all three schemes seem to have the same pre-log constant as the AWGN capacity at high average SNR. All these observations corroborate our analysis in Section IV. Figure 2 shows the capacity differences at finite average SNRs. We observe that for average SNR higher than $6 \mathrm{~dB}$, the gap between OA and CI becomes almost steady at the value 0.24928 (bits) also predicted by our analytical results in (47). However, the value 0.45943 obtained through (52) which is the asymptotic gap between AWGN and CI requires significantly higher average SNR (about $40 \mathrm{~dB}$ ) to become visible.

Figure 3 shows the capacity curves of two TCI schemes with different fixed thresholds as well as CI for the case of $(N, K)=(2,2)$. We observe that TCI exhibits a smaller pre-log constant than CI at high average SNR, and a larger threshold results in a smaller pre-log constant. Consequently, TCI has worse capacity than CI at high average SNR, as expected. However, the conclusion is reversed at sufficiently low average SNR: TCI gives better capacity than CI, and a larger threshold is more favorable. This corroborates our analysis in Subsections IV-A and IV-D. In Figure 4 we compare TCI with optimal threshold and CI for $(N, K)=(1,4)$ and $(2,2)$. Figure 5 shows the optimal thresholds of TCI at different average SNRs for $(N, K)=(1,4)$ and $(2,2)$. We observe that as the average SNR increases, the optimal threshold of TCI becomes 
smaller from Figure 5. Consequently, the capacity of TCI becomes closer to the capacity of CI at higher average SNR in both choices of $(N, K)$, as can be observed from Figure 4. These observations corroborate our analysis in IV-A.

In Figure 6 we give comparisons of TCI, OA and AWGN, at low average SNR. We observe that both OA and TCI can provide better capacity than the AWGN case, which verifies our analysis in Subsection IV-D. In addition, we mentioned in Subsection IV-D that $z_{\mathrm{t}} \geq \mathrm{E}\left[z_{\mathrm{eff}}\right]$ is sufficient for TCI to have better capacity than AWGN. However for $(N, K)=(2,2)$ we have $\mathrm{E}\left[z_{\text {eff }}\right]=2.75>2.5$, which indicates that $z_{\mathrm{t}} \geq \mathrm{E}\left[z_{\text {eff }}\right]$ is not actually necessary. Since TCI has significantly less implementation complexity than OA, it is shown to be a viable adaptive transmission scheme at low average SNR.

Figure 7 shows the asymptotic gap between OA and CI obtained from (47) and the asymptotic gap between AWGN and CI obtained from (52), for $K=1$ and different values of $N$. We observe that the gaps are both decreasing and converging to zero with the increase of diversity order, and appear to be inversely proportional to $N-1$ since the plots become almost straight lines with slope -1 , as suggested by (55) and (56). Figure 8 shows the same thing depicted by Figure 7 for $N=1$ and different values of $K$. It also displays the fact that the gaps become smaller and converge to zero as the number of users increases, but the decrease is much slower than the case of Figure 7 since the gap between AWGN and CI is inversely proportional to $\log K$.

\section{CONCLUSIONS}

In this paper we investigate asymptotic properties at both high and low average SNRs of the ergodic capacities for several adaptive transmission schemes and a wide class of channel distributions. We show that at high average SNR, both CI and CTCI exhibit the same capacity pre-log constant of 1 , while TCI exhibits a pre-log constant which is strictly less than 1 . This is a special case of a more general result (Theorem 2) which shows that the presence of outage in the high-SNR instantaneous power ratio reduces the pre-log constant. Consequently, with the average SNR being sufficiently high, both CI and CTCI outperform TCI. In addition, we prove that the capacity of CTCI is monotonically increasing with the threshold for any value of average SNR, which is used to show that the largest asymptotic capacity gap among all the schemes under fading is the one between CI and OA. We have derived closed-form expressions for asymptotic gaps between $\mathrm{CI}$ and $\mathrm{OA}$ as well as $\mathrm{CI}$ and AWGN. For the case of low average 
SNR, we show that AWGN capacity can be exceeded by OA and TCI. Consequently, TCI is a favorable scheme at low average SNR since it has significantly less complexity than OA.

We also study the behavior of the derived high-SNR gaps among CI, OA and AWGN in the presence of diversity. Through the examples of space diversity and multi-user diversity, we point out that the high-SNR gaps can be made arbitrarily small with sufficiently large number of antennas or users. Based on our expressions for rates of convergence, it is shown that antennas are more efficient than users in reducing the gaps. An example of a channel distribution under which the sub-optimality gaps are independent of $K$ is also given to illustrate that the gaps need not always reduce with $K$. This indicates that under certain conditions CI is asymptotically optimal with sufficiently large diversity order.

\section{APPENDIX I}

\section{IMPLICATIONS OF A1}

In this appendix, we show that $\mathbf{A 1}$ implies $F_{\gamma}(0)=0$ and $|\mathrm{E}[\log \gamma]|<\infty$. Since $F_{\gamma}(x)$ is regularly varying at the origin, we have $\lim _{x \rightarrow 0} F_{\gamma}(\tau x) / F_{\gamma}(x)=\tau^{d}$ for $\tau>0$. Clearly, as $\tau \rightarrow 0$ it is required that $F_{\gamma}(0) / F_{\gamma}(x)=0$ since $d>0$. Moreover since $0 \leq F_{\gamma}(x) \leq 1$, we obtain $F_{\gamma}(0)=0$.

We next prove that $\mathrm{E}[|\log \gamma|]<\infty$, which implies that $|\mathrm{E}[\log \gamma]|<\mathrm{E}[|\log \gamma|]<\infty$. Define $\delta=\sup \left\{x: F_{\gamma}(x)<1\right\}$. We consider the cases of $0<\delta \leq 1$ and $\delta>1$. We will use for both cases, since $\log x$ is slowly varying at $x=0, \log x F_{\gamma}(x)$ is regularly varying with exponent $d>0$ similar to $F_{\gamma}(x)$, thus $\lim _{x \rightarrow 0} \log x F_{\gamma}(x)=0$.

If $0<\delta \leq 1$, we have $\mathrm{E}[|\log \gamma|]=-\int_{0}^{\delta} \log x f_{\gamma}(x) d x=\int_{0}^{\delta} x^{-1} F_{\gamma}(x) d x-\log \delta$ using integration by parts and $\lim _{x \rightarrow 0} \log x F_{\gamma}(x)=0$. Let $y=x^{-1}, l(x)=r\left(x^{-1}\right)=r(y)$ and $F_{\gamma}(x)=G\left(x^{-1}\right)=G(y)$, we have $\int_{0}^{\delta} x^{-1} F_{\gamma}(x) d x=\int_{1 / \delta}^{\infty} y^{-1} G(y) d y=\int_{1 / \delta}^{\infty} y^{-1-d} r(y) d y$, and $r(y)$ varies slowly at $\infty$. Consequently, we have $\int_{0}^{\delta} x^{-1} F_{\gamma}(x) d x=\int_{1 / \delta}^{\infty} y^{-1-d} r(y) d y<\infty$, which can be justified by a modification of the Lemma in [14, pp.280] given $-1-d<-1$. It follows that $\mathrm{E}[|\log \gamma|]=\int_{0}^{\delta} x^{-1} F_{\gamma}(x) d x-\log \delta<\infty$.

If $\delta>1$, similar to the previous case, we have $\mathrm{E}[|\log \gamma|]=\int_{0}^{1} x^{-1} F_{\gamma}(x) d x+\int_{1}^{\delta} \log x f_{\gamma}(x) d x$, and it can be proved that $\int_{0}^{1} x^{-1} F_{\gamma}(x) d x<\infty$. Furthermore, since $\log x \leq x-1, \int_{1}^{\delta} \log x f_{\gamma}(x) d x \leq$ $\int_{1}^{\delta}(x-1) f_{\gamma}(x) d x<\mathrm{E}[\gamma]+F_{\gamma}(1)-1<\infty$. It follows that $\mathrm{E}[|\log \gamma|]<\infty$. 


\section{APPENDIX II}

\section{PROOF OF LEMMA 1}

We first show that $\limsup _{S \rightarrow 0} z_{\mathrm{t}}=\sup \left\{z: F_{z_{\text {eff }}}(z)<1\right\}$. If instead, there exists $\delta$ such that $z_{\mathrm{t}} \leq \delta<\sup \left\{z: F_{z_{\mathrm{eff}}}(z)<1\right\}$ for all $S$ then

$$
\frac{1}{S} \int_{\delta}^{\infty}\left(\frac{1}{\delta}-\frac{1}{z}\right) f_{z_{\mathrm{eff}}}(z) d z \leq \int_{z_{\mathrm{t}}}^{\infty} \frac{1}{S}\left(\frac{1}{z_{\mathrm{t}}}-\frac{1}{z}\right) f_{z_{\mathrm{eff}}}(z) d z
$$

since the left hand side of (19) is monotonically decreasing with $z_{\mathrm{t}}$ for a fixed $S$. However, (60) cannot hold since as $S \rightarrow 0$ it violates (19). Moreover since from (23) $z_{\mathrm{t}}$ is monotonically decreasing with $S$, we have $\lim _{S \rightarrow 0} z_{\mathrm{t}}=\sup \left\{z: F_{z_{\text {eff }}}(z)<1\right\}$. It can be seen from (22) that $0 \leq z_{\mathrm{t}}<1 / S$, and consequently $\lim _{S \rightarrow \infty} z_{\mathrm{t}}=0$.

\section{APPENDIX III}

\section{Proof OF TheOREM 1}

Differentiating (35) with respect to $z_{\mathrm{t}}$, we obtain

$$
\begin{aligned}
\frac{d C_{\mathrm{CTCI}}}{d z_{\mathrm{t}}} & =\int_{0}^{z_{\mathrm{t}}} \frac{-S D_{\mathrm{max}}^{2} z}{1+S D_{\max } z}\left(\int_{z_{\mathrm{t}}}^{\infty} \frac{1}{z} f_{z_{\mathrm{eff}}}(z) d z\right) f_{z_{\mathrm{eff}}}(z) d z \\
& +\frac{1-F_{z_{\mathrm{eff}}}\left(z_{\mathrm{t}}\right)}{1+S D_{\max } z_{\mathrm{t}}}\left(S D_{\max }-S D_{\max }^{2} z_{\mathrm{t}} \int_{z_{\mathrm{t}}}^{\infty} \frac{1}{z} f_{z_{\mathrm{eff}}}(z) d z\right) \\
& =F_{z_{\mathrm{eff}}}\left(z_{\mathrm{t}}\right)\left[1-F_{z_{\mathrm{eff}}}\left(z_{\mathrm{t}}\right) \frac{S D_{\max }^{2}}{1+S D_{\max } z_{\mathrm{t}}}\right. \\
& -\left(\int_{z_{\mathrm{t}}}^{\infty} \frac{1}{z} f_{z_{\mathrm{eff}}}(z) d z\right)\left(\int_{0}^{z_{\mathrm{t}}} \frac{S D_{\mathrm{max}}^{2} z}{1+S D_{\max } z} f_{z_{\mathrm{eff}}}(z) d z\right)
\end{aligned}
$$

Notice that we take $D_{\max }$ as a function of $z_{\mathrm{t}}$ given by (34) in (61). It is easy to prove that $S D_{\max }^{2} z /\left(1+S D_{\max } z\right)$ is monotonically increasing with $z$, and $1 / z$ is monotonically decreasing with $z$, therefore

$$
\begin{gathered}
\int_{0}^{z_{\mathrm{t}}} \frac{S D_{\max }^{2} z}{1+S D_{\max } z} f_{z_{\mathrm{eff}}}(z) d z<\int_{0}^{z_{\mathrm{t}}} \frac{S D_{\max }^{2} z_{\mathrm{t}}}{1+S D_{\max } z_{\mathrm{t}}} f_{z_{\mathrm{eff}}}(z) d z=\frac{S D_{\max }^{2} z_{\mathrm{t}}}{1+S D_{\max } z_{\mathrm{t}}} F_{z_{\mathrm{eff}}}\left(z_{\mathrm{t}}\right) \\
\int_{z_{\mathrm{t}}}^{\infty} \frac{1}{z} f_{z_{\mathrm{eff}}}(z) d z<\int_{z_{\mathrm{t}}}^{\infty} \frac{1}{z_{\mathrm{t}}} f_{z_{\mathrm{eff}}}(z) d z=\frac{1-F_{z_{\mathrm{eff}}}\left(z_{\mathrm{t}}\right)}{z_{\mathrm{t}}}
\end{gathered}
$$

Multiplying (62a) with (62b) then substituting the result into (61), we obtain $d C_{\mathrm{CTCI}} / d z_{\mathrm{t}}>0$, i.e. $C_{\mathrm{CTCI}}$ is monotonically increasing with $z_{\mathrm{t}}$ regardless of $S$. 


\section{APPENDIX IV}

\section{Proof of TheOREM 2}

Let $C=\mathrm{E}\left[\log \left(1+S D\left(z_{\mathrm{eff}}\right) z_{\mathrm{eff}}\right)\right]=\int_{0}^{\infty} \log (1+S D(z) z) f_{z_{\mathrm{eff}}}(z) d z$ and denote the complementary set of $\mathscr{O}=\{z \mid D(z)=0\}$ on $[0, \infty)$ by $\overline{\mathscr{O}}$. We have

$$
\frac{d C}{d(\log S)}=S \frac{d C}{d S}=\int_{0}^{\infty}\left(\frac{S D(z) z}{1+S D(z) z}\right) f_{z_{\mathrm{eff}}}(z) d z
$$

Clearly, $\lim _{S \rightarrow \infty}(S D(z) z) /(1+S D(z) z)$ becomes 1 with $D(z) \neq 0$ and 0 with $D(z)=0$, and the limit and integral can be exchanged since the absolute value of the integrand is upper bounded by $f_{z_{\text {eff }}}(z)$, which is an integrable function. Therefore the limit of (63) as $S \rightarrow \infty$ becomes

$$
\begin{aligned}
\lim _{S \rightarrow \infty} \frac{d C}{d(\log S)} & =\int_{\overline{\mathscr{O}}} 1 \cdot f_{z_{\mathrm{eff}}}(z) d z+\int_{\mathscr{O}} 0 \cdot f_{z_{\mathrm{eff}}}(z) d z=\int_{0}^{\infty} f_{z_{\mathrm{eff}}}(z) d z-\int_{\mathscr{O}} f_{z_{\mathrm{eff}}}(z) d z+0 \\
& =1-\operatorname{Pr}(\mathscr{O}) .
\end{aligned}
$$

\section{APPENDIX V}

\section{Proof of LeMma 2}

With $\mu$ being a positive constant, $\log ((1+S \mu) /(S \mu))$ is independent of $z$, therefore

$$
\lim _{S \rightarrow \infty} \int_{0}^{\infty} \log \left(\frac{1+S \mu}{S \mu}\right) f_{z_{\mathrm{eff}}}(z) d z=\lim _{S \rightarrow \infty} \log \left(\frac{1+S \mu}{S \mu}\right) \cdot \int_{0}^{\infty} f_{z_{\mathrm{eff}}}(z) d z=\log 1 \cdot 1=0
$$

Since $\log ((1+S z) / S)=\log (1 / S+z)$ is monotonically decreasing with $S$, it is easy to show that $\int_{0}^{\infty} \log ((1+S z) / S) f_{z_{\text {eff }}}(z) d z$ is monotonically decreasing with $S$ and has an infimum of $\int_{0}^{\infty} \log z f_{z_{\mathrm{eff}}}(z) d z$ as $S \rightarrow \infty$. Consequently, due to monotone convergence theorem, which indicates that the limit of a sequence of real numbers is its infimum if it is decreasing and bounded below, we have

$$
\begin{aligned}
\lim _{S \rightarrow \infty} \int_{0}^{\infty} \log \left(\frac{1+S z}{S}\right) f_{z_{\mathrm{eff}}}(z) d z & =\inf \left\{\int_{0}^{\infty} \log \left(\frac{1+S z}{S}\right) f_{z_{\mathrm{eff}}}(z) d z: S>0\right\} \\
& =\int_{0}^{\infty} \log z f_{z_{\mathrm{eff}}}(z) d z
\end{aligned}
$$

and therefore

$$
\lim _{S \rightarrow \infty} \int_{0}^{\infty} \log \left(\frac{1+S z}{S z}\right) f_{z_{\mathrm{eff}}}(z) d z=\lim _{S \rightarrow \infty} \int_{0}^{\infty} \log \left(\frac{1+S z}{S}\right) f_{z_{\mathrm{eff}}}(z) d z-\int_{0}^{\infty} \log z f_{z_{\mathrm{eff}}}(z) d z=0
$$




\section{APPENDIX VI}

\section{ProOF of TheOREM 3}

We first prove (45), which will be useful for subsequent derivations since the gaps of OA and RA with respect to a third scheme become equivalent. From (37) and (20) we obtain

$$
\begin{aligned}
C_{\mathrm{OA}}-C_{\mathrm{RA}} & \leq \int_{z_{\mathrm{t}}}^{\infty} \log \left(\frac{z}{z_{\mathrm{t}}}\right) f_{z_{\mathrm{eff}}}(z) d z-\int_{z_{\mathrm{t}}}^{\infty} \log (1+S z) f_{z_{\mathrm{eff}}}(z) d z \\
& =\int_{z_{\mathrm{t}}}^{\infty} \log \left(\frac{z}{z_{\mathrm{t}}+S z_{\mathrm{t}} z}\right) f_{z_{\mathrm{eff}}}(z) d z<\int_{z_{\mathrm{t}}}^{\infty} \log \left(\frac{z+S^{-1}}{z_{\mathrm{t}}+S z_{\mathrm{t}} z}\right) f_{z_{\mathrm{eff}}}(z) d z \\
& =\int_{z_{\mathrm{t}}}^{\infty} \log \left(\frac{1}{S z_{\mathrm{t}}}\right) f_{z_{\mathrm{eff}}}(z) d z=-\log \left(S z_{\mathrm{t}}\right)\left(1-F_{z_{\mathrm{eff}}}\left(z_{\mathrm{t}}\right)\right)
\end{aligned}
$$

Since the OA threshold satisfies $\lim _{S \rightarrow \infty} z_{\mathrm{t}}=0$ by Lemma $1, \lim _{S \rightarrow \infty} S z_{\mathrm{t}}=\lim _{S \rightarrow \infty} d C_{\mathrm{OA}} / d(\log S)=$ 1 based on (25). Furthermore, $C_{\mathrm{OA}}-C_{\mathrm{RA}} \geq 0$ due to the optimality of OA. Taking the limit in (68), we obtain (45). This indicates that RA is asymptotically optimal with sufficiently high average SNR.

We now use Lemma 2 and (45) to show (46)

$$
\begin{aligned}
\lim _{S \rightarrow \infty}\left(C_{\mathrm{AWGN}}-C_{\mathrm{OA}}\right) & =\lim _{S \rightarrow \infty}\left(C_{\mathrm{AWGN}}-C_{\mathrm{RA}}\right)=\lim _{S \rightarrow \infty} \int_{0}^{\infty} \log \left(\frac{1+S \mathrm{E}\left[z_{\mathrm{eff}}\right]}{1+S z}\right) f_{z_{\mathrm{eff}}}(z) d z \\
& =\lim _{S \rightarrow \infty} \int_{0}^{\infty} \log \left(\frac{1+S \mathrm{E}\left[z_{\mathrm{eff}}\right]}{1+S z}\right) f_{z_{\mathrm{eff}}}(z) d z+\lim _{S \rightarrow \infty} \int_{0}^{\infty} \log \left(\frac{1+S z}{S z}\right) f_{z_{\mathrm{eff}}}(z) d z \\
& +\lim _{S \rightarrow \infty} \int_{0}^{\infty} \log \left(\frac{S \mathrm{E}\left[z_{\mathrm{eff}}\right]}{1+S \mathrm{E}\left[z_{\mathrm{eff}}\right]}\right) f_{z_{\mathrm{eff}}}(z) d z \\
& =\int_{0}^{\infty} \log \left(\frac{\mathrm{E}\left[z_{\mathrm{eff}}\right]}{z}\right) f_{z_{\mathrm{eff}}}(z) d z=\log \left(\mathrm{E}\left[z_{\mathrm{eff}}\right]\right)-\mathrm{E}\left[\log z_{\mathrm{eff}}\right]
\end{aligned}
$$

where we use (44) in the second term, and (43) with $\mu=\mathrm{E}\left[z_{\text {eff }}\right]$ in the third term following the third equality (marked " $\nmid$ "). Notice that (46) is guaranteed to be finite when A1 holds, since it ensures $C_{\mathrm{AWGN}}$ and $C_{\mathrm{OA}}$ have the same high-SNR pre-log constant, and also a finite $\mathrm{E}\left[\log z_{\text {eff }}\right]$. Moreover, we have $\log \left(\mathrm{E}\left[z_{\mathrm{eff}}\right]\right) \geq \mathrm{E}\left[\log z_{\text {eff }}\right]$ based on Jensen's inequality and thus (46) is non-negative. 
To show (47), using a similar approach as in (69), we obtain

$$
\begin{aligned}
\lim _{S \rightarrow \infty}\left(C_{\mathrm{OA}}-C_{\mathrm{CI}}\right) & =\lim _{S \rightarrow \infty}\left(C_{\mathrm{RA}}-C_{\mathrm{CI}}\right)=\lim _{S \rightarrow \infty}\left(\int_{0}^{\infty} \log (1+S z) f_{z_{\mathrm{eff}}}(z) d z-\log \left(1+S \mathrm{E}^{-1}\left[z_{\text {eff }}^{-1}\right]\right)\right) \\
& =\lim _{S \rightarrow \infty} \int_{0}^{\infty} \log \left(\frac{1+S z}{1+S \mathrm{E}^{-1}\left[z_{\mathrm{eff}}^{-1}\right]}\right) f_{z_{\mathrm{eff}}}(z) d z=^{\ddagger} \int_{0}^{\infty} \log \left(\frac{z}{\mathrm{E}^{-1}\left[z_{\mathrm{eff}}^{-1}\right]}\right) f_{z_{\mathrm{eff}}}(z) d z \\
& =\mathrm{E}\left[\log z_{\mathrm{eff}}\right]+\log \left(\mathrm{E}\left[z_{\text {eff }}^{-1}\right]\right)
\end{aligned}
$$

where the first equality (marked "†") is based on (45), and the fourth equality (marked "†") is based on Lemma 2 with $\mu=\mathrm{E}^{-1}\left[z_{\text {eff }}^{-1}\right]$. Similar to (46), (47) is non-negative due to Jensen's inequality, and it is finite since both $\mathrm{E}\left[\log z_{\mathrm{eff}}\right]$ and $\log \left(\mathrm{E}\left[z_{\mathrm{eff}}^{-1}\right]\right)$ are finite given $\mathbf{A 2}$.

\section{APPENDIX VII \\ PROOF OF THEOREM 4}

Since $C_{\mathrm{CI}} \leq C_{\mathrm{CTCI}} \leq C_{\mathrm{RA}}$ for any $S$, and all capacities are zero at $S=0$, the inequalities in (49) hold, and the first and last equalities follow from (39) and (40). (50) can be obtained based on the derivation after Lemma 1 in IV-A, and the fact that the least upper bound is no less than the mean. (51) holds since (32) indicates that $\lim _{S \rightarrow 0} d C_{\mathrm{TCI}} / d S$ is monotonically increasing with $z_{\mathrm{t}}$. In addition, $\lim _{S \rightarrow 0} d C_{\mathrm{TCI}} / d S \geq z_{\mathrm{t}}$ as can be obtained through upper-bounding the denominator in (32) by $\int_{z_{\mathrm{t}}}^{\infty} z_{\mathrm{t}}^{-1} f_{z_{\mathrm{eff}}}(z) d z$, therefore $z_{\mathrm{t}} \geq \mathrm{E}\left[z_{\mathrm{eff}}\right]$ is a sufficient condition for $\lim _{S \rightarrow 0} d C_{\mathrm{TCI}} / d S \geq \lim _{S \rightarrow 0} d C_{\mathrm{AWGN}} / d S$.

\section{REFERENCES}

[1] A. J. Goldsmith and P. P. Varaiya, "Capacity of fading channels with channel side information," IEEE Transactions on Information Theory, vol. 43, no. 6, pp. 1986-1992, Nov. 1997.

[2] A. Laourine, M.-S. Alouini, S. Affes, and A. Stephenne, "On the capacity of generalized-k fading channels," IEEE Transactions on Wireless Communications, vol. 7, no. 7, pp. 2441-2445, Jul. 2008.

[3] — _ "On the performance analysis of composite multipath/shadowing channels using the G-distribution," IEEE Transactions on Communications, vol. 57, no. 4, pp. 1162-1170, Apr. 2009.

[4] G. Song and Y. Li, “Asymptotic throughput analysis of multiuser diversity," IEEE Global Telecommunications Conference, vol. 3, pp. 1289-1293, Nov. 2005.

[5] M.-S. Alouini and A. J. Goldsmith, "Capacity of Nakagami multipath fading channels," IEEE 47th Vehicular Technology Conference, vol. 1, pp. 358-362, May. 1997.

[6] - "Capacity of Rayleigh fading channels under different adaptive transmission and diversity-combining techniques," IEEE Transactions on Vehicular Technology, vol. 48, no. 4, pp. 1165-1181, Jul. 1999. 
[7] J. W. Shao, M.-S. Alouini, and A. J. Goldsmith, "Impact of fading correlation and unequal branch gains on the capacity of diversity systems," IEEE 49th Vehicular Technology Conference, vol. 3, pp. 2159-2163, May. 1999.

[8] P. S. Bithas and P. T. Mathiopoulos, "Capacity of Correlated Generalized Gamma Fading With Dual-Branch Selection Diversity,” IEEE Transactions on Vehicular Technology, vol. 58, no. 9, pp. 5258-5663, Nov. 2009.

[9] R. K. Mallik, M. Z. Win, J. W. Shao, M.-S. Alouini, and A. J. Goldsmith, "Channel capacity of adaptive transmission with maximal ratio combining in correlated Rayleigh fading," IEEE Transactions on Wireless Communications, vol. 3, no. 4, pp. 1124-1133, Jul. 2004.

[10] S. Khatalin and J. P. Fonseka, "Channel capacity of dual-branch diversity systems over correlated Nakagami-m fading with channel inversion and fixed rate transmission scheme," IET Communications, vol. 1, pp. 1161-1169, Dec. 2007.

[11] Z. M. Hussain, S. A. Zummo, F. S. Al-Qahtani, and A. K. Gurung, "Spectral efficiency evaluation for selection combining diversity (SCD) scheme over slow fading," IET Communications, vol. 3, pp. 1443-1451, Sep. 2009.

[12] A. J. Goldsmith and S.-G. Chua, "Variable-rate variable-power MQAM for fading channels," IEEE Transactions on Communications, vol. 45, no. 10, pp. 1218-1230, Oct. 1997.

[13] S. Vishwanath and A. J. Goldsmith, "Adaptive turbo-coded modulation for flat-fading channels," IEEE Transactions on Communications, vol. 51, no. 6, pp. 964-972, Jun. 2003.

[14] W. Feller, An Introduction to Probability Theory and Its Applications: Volume II, 2nd ed. New York: John Wiley and Sons, 1971.

[15] Z. Wang and G. B. Giannakis, "A simple and general parameterization quantifying performance in fading channels," IEEE Transactions on Communications, vol. 51, no. 8, pp. 1389-1398, Aug. 2003.

[16] R. McEliece and W. Stark, "Channels with block interference," IEEE Transactions on Information Theory, vol. 30, no. 1, pp. 44-53, Jan. 1984.

[17] A. Lapidoth, "On the asymptotic capacity of stationary Gaussian fading channels," IEEE Transactions on Information Theory, vol. 51, no. 2, pp. 437-446, Feb. 2005.

[18] M.-S. Alouini and A. J. Goldsmith, "Comparison of fading channel capacity under different CSI assumptions," IEEE 52nd Vehicular Technology Conference, vol. 4, pp. 1844-1849, Sep 2000.

[19] D. N. C. Tse and P. Viswanath, Fundamentals of Wireless Communication, 1st ed. Cambridge: Cambridge University Press, Jun. 2005.

[20] E. Biglieri, J. Proakis, and S. Shamai, "Fading Channels: Information-Theoretic and Communications Aspects," IEEE Transactions on Information Theory, vol. 44, no. 6, pp. 2619-2692, Oct. 1998.

[21] A. S. Leong and S. Dey, "On Scaling Laws of Diversity Schemes in Decentralized Estimation," accepted for publication on IEEE Transactions on Information Theory, also available online at http://arxiv.org/abs/1002.4473.

[22] A. DasGupta, Asymptotic Theory of Statistics and Probability, 1st ed. Springer, Mar. 2008.

[23] M.-O. Pun, V. Koivunen, and H. V. Poor, "Opportunistic Scheduling and Beamforming for MIMO-SDMA Downlink Systems with Linear Combining," IEEE 18th International Symposium on Personal, Indoor and Mobile Radio Communications, pp. 1-6, Sep. 2007. 


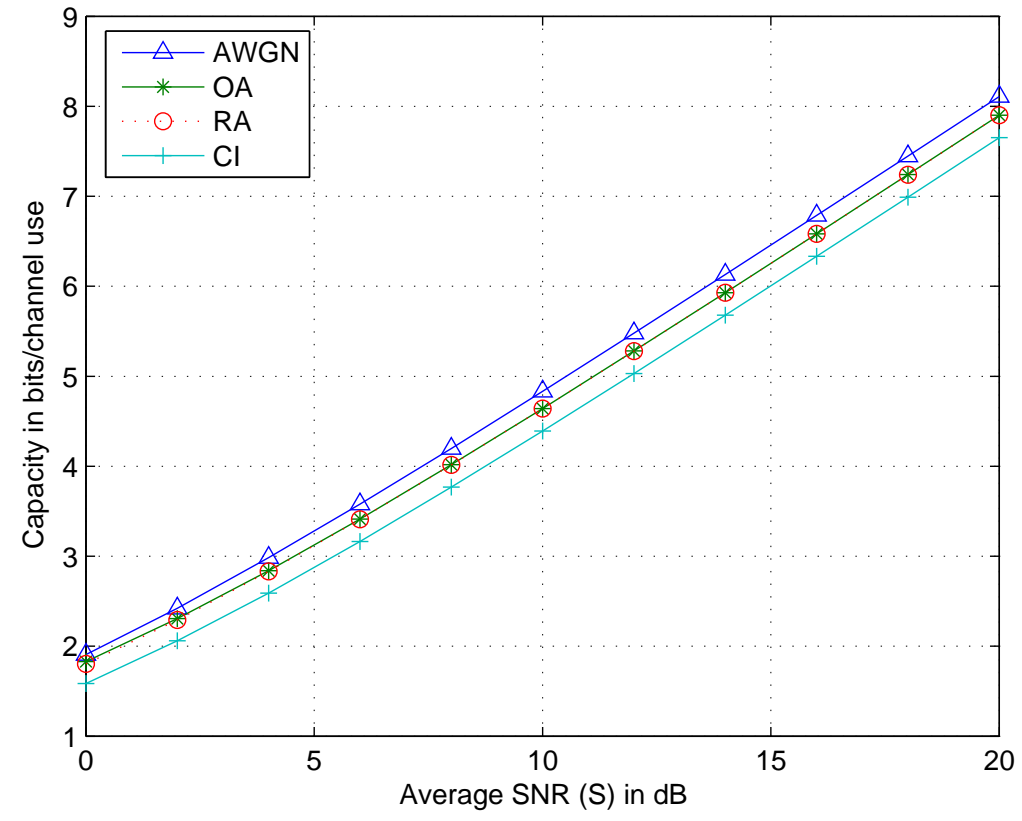

Fig. 1. Capacities of AWGN, OA, RA and CI for $(N, K)=(2,2)$

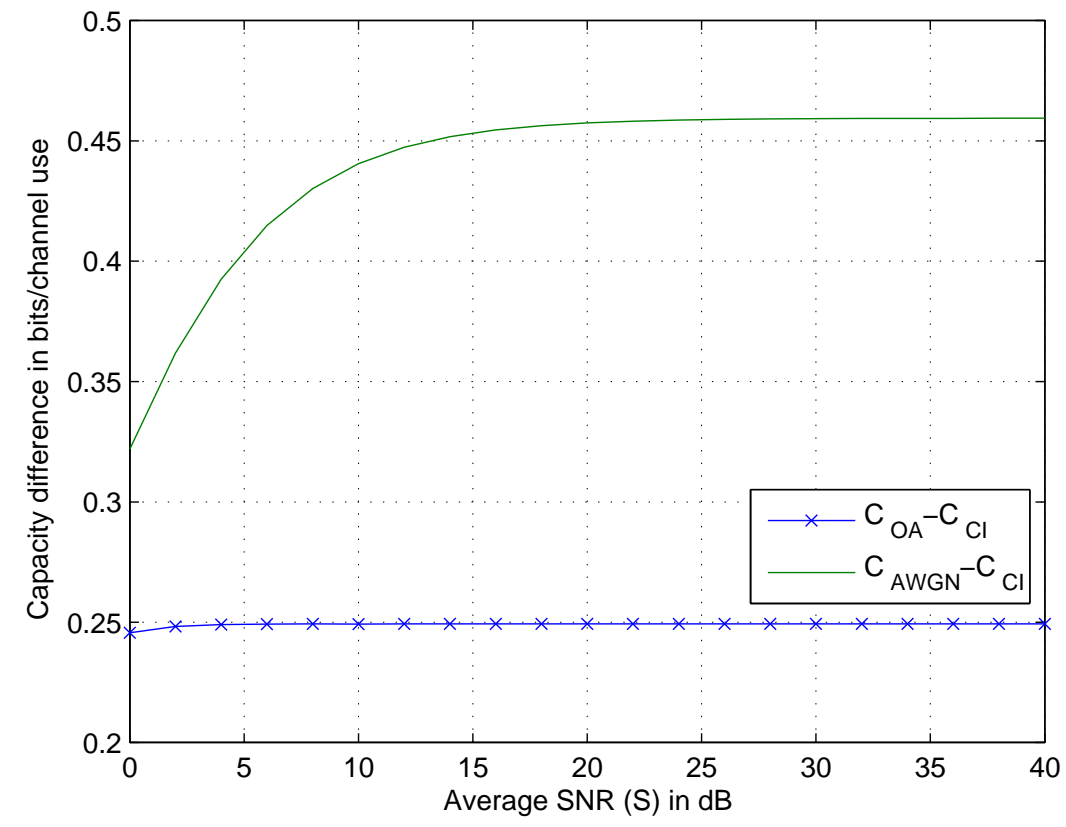

Fig. 2. Capacity differences from OA and AWGN to CI at different average SNRs for $(N, K)=(2,2)$ 


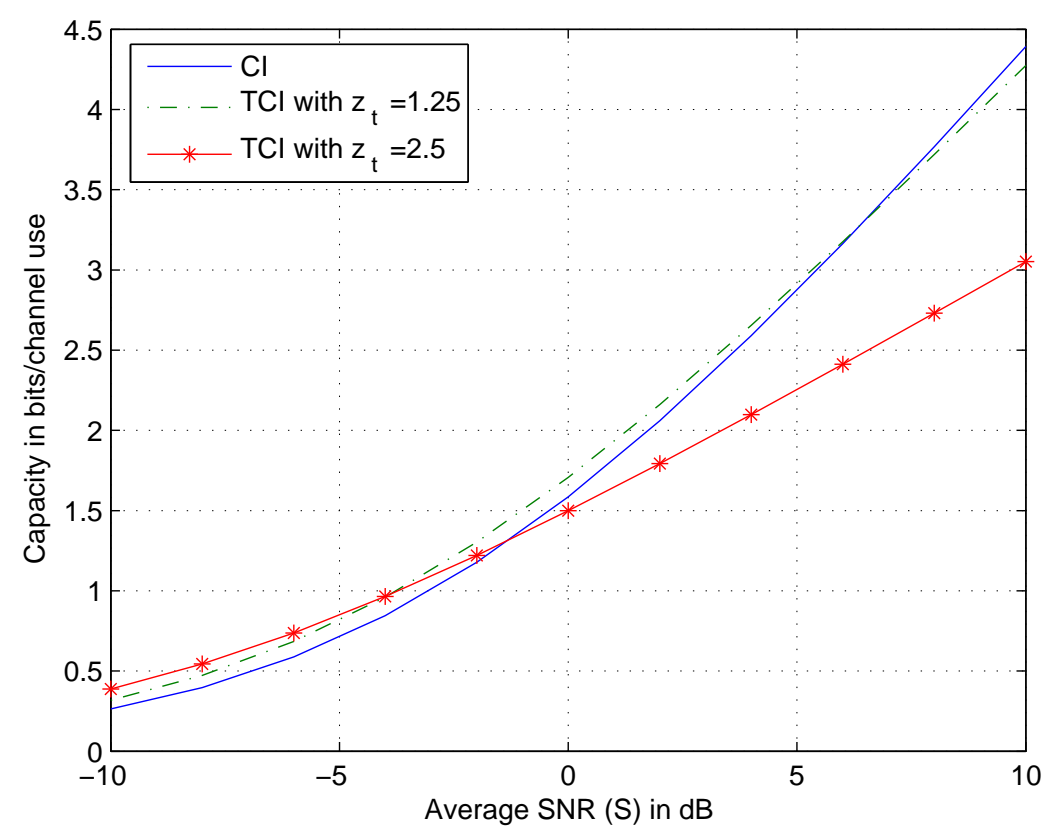

Fig. 3. Capacities of CI and TCI for $(N, K)=(2,2)$

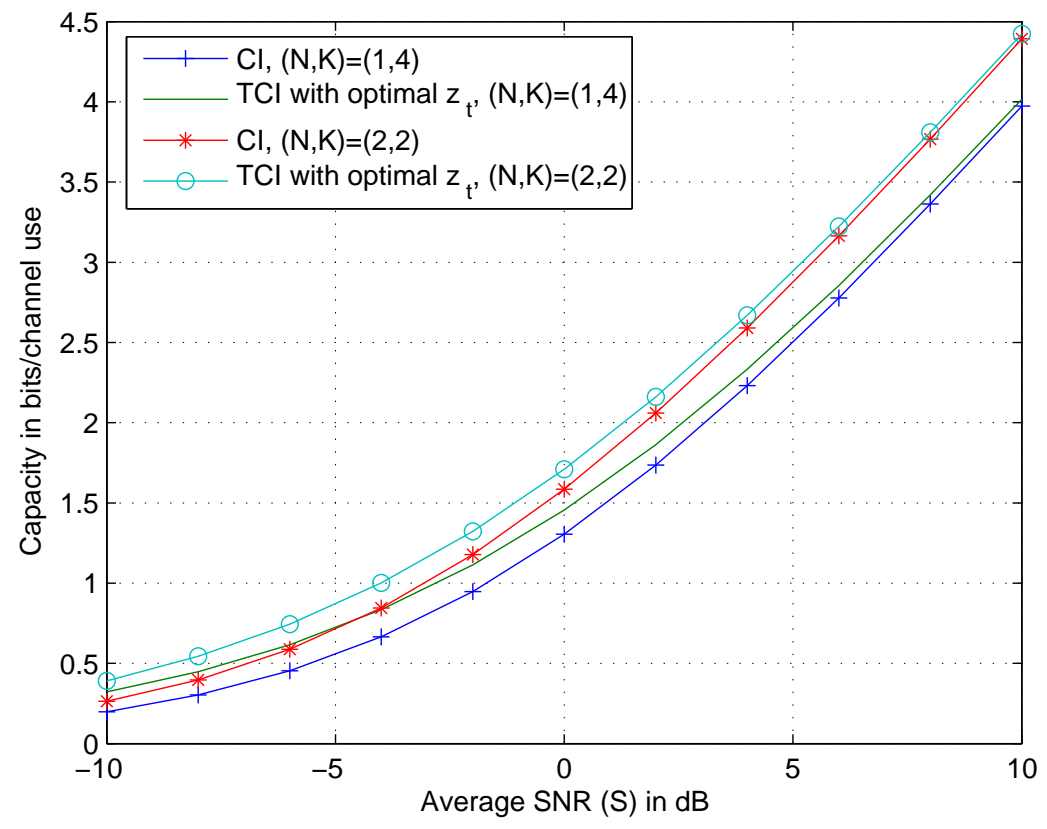

Fig. 4. Capacities of CI and optimized TCI for $(N, K)=(1,4)$ and $(2,2)$ 


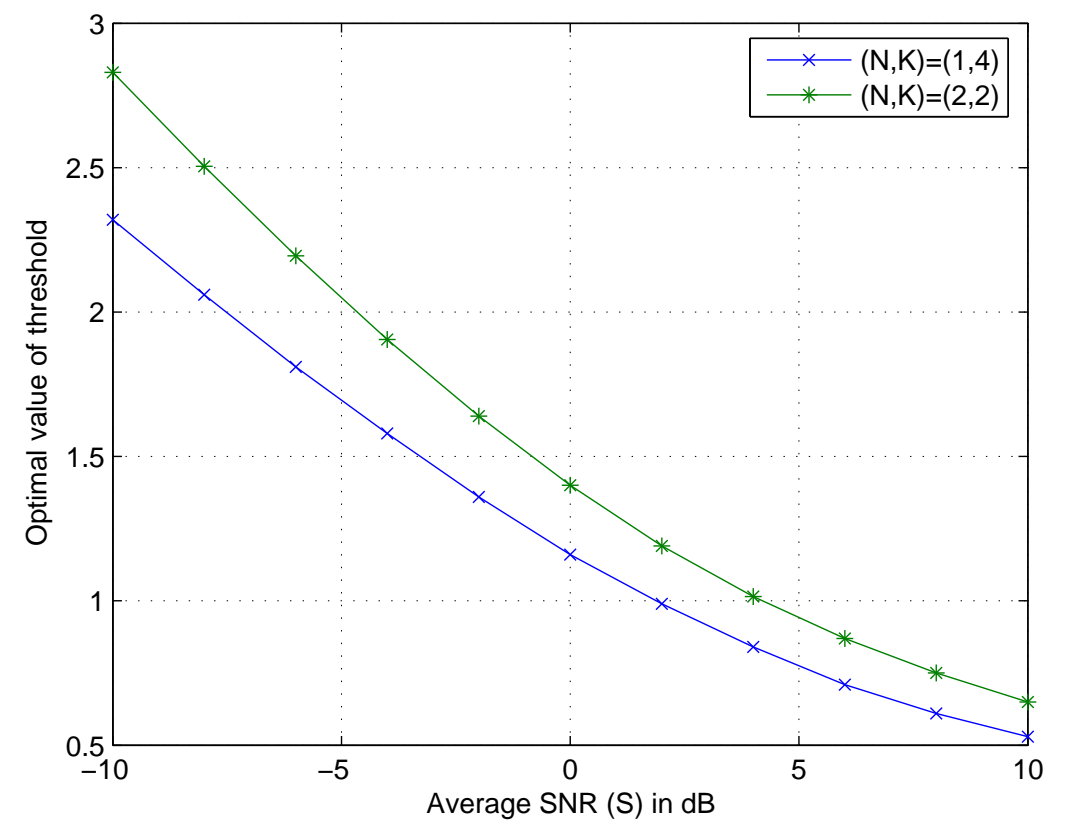

Fig. 5. Optimal $z_{\mathrm{t}}$ of TCI for $(N, K)=(1,4)$ and $(2,2)$

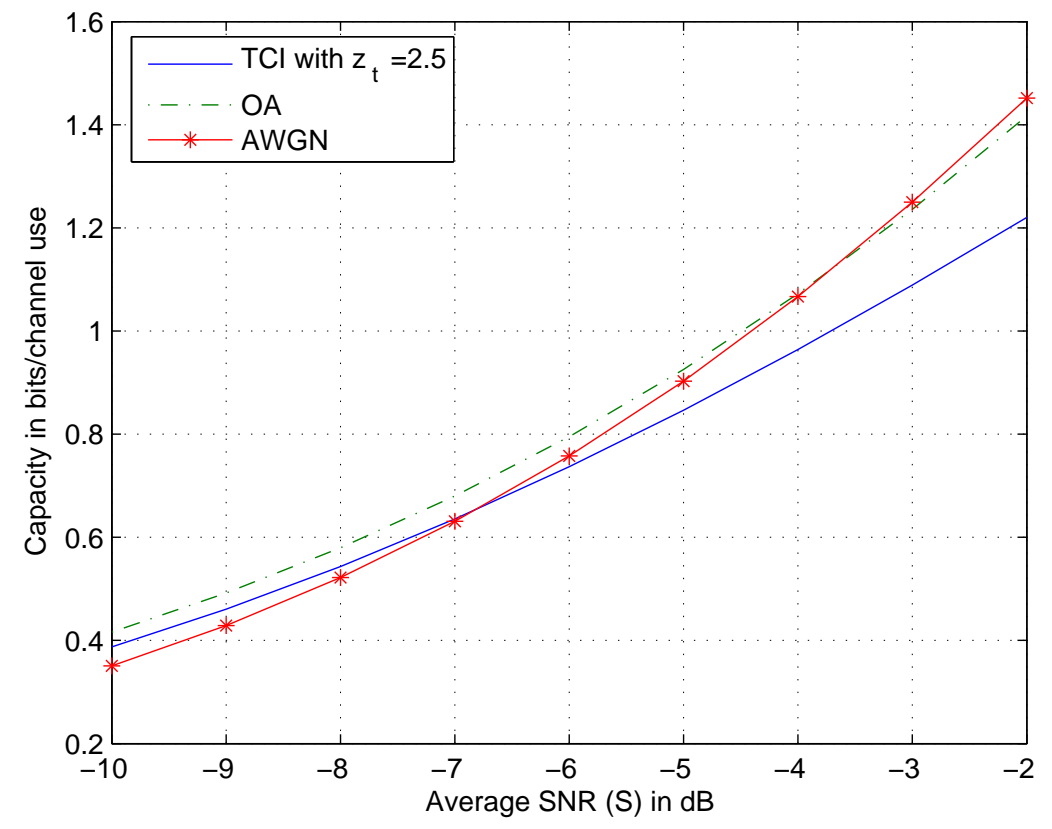

Fig. 6. Capacities of TCI, OA and AWGN for $(N, K)=(2,2)$ at low average SNR 


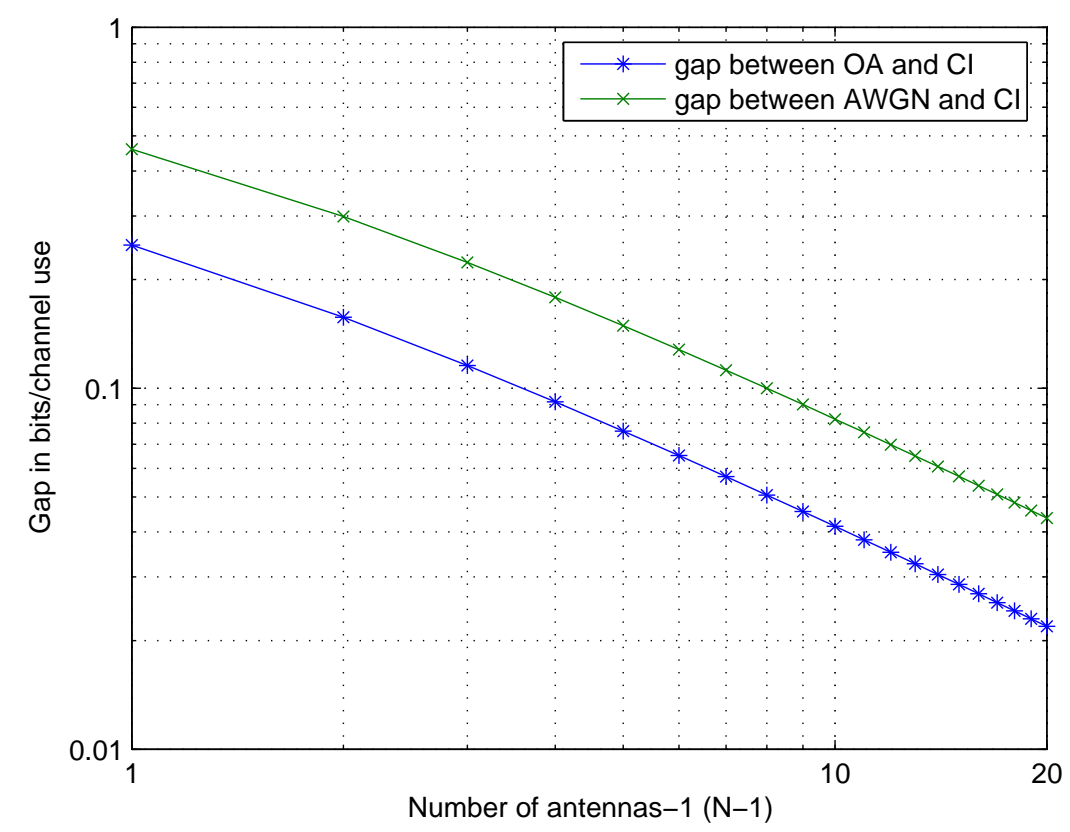

Fig. 7. $\lim _{S \rightarrow \infty}\left(C_{\mathrm{OA}}-C_{\mathrm{CI}}\right)$ and $\lim _{S \rightarrow \infty}\left(C_{\mathrm{AWGN}}-C_{\mathrm{CI}}\right)$ with transmit or receive diversity between two points

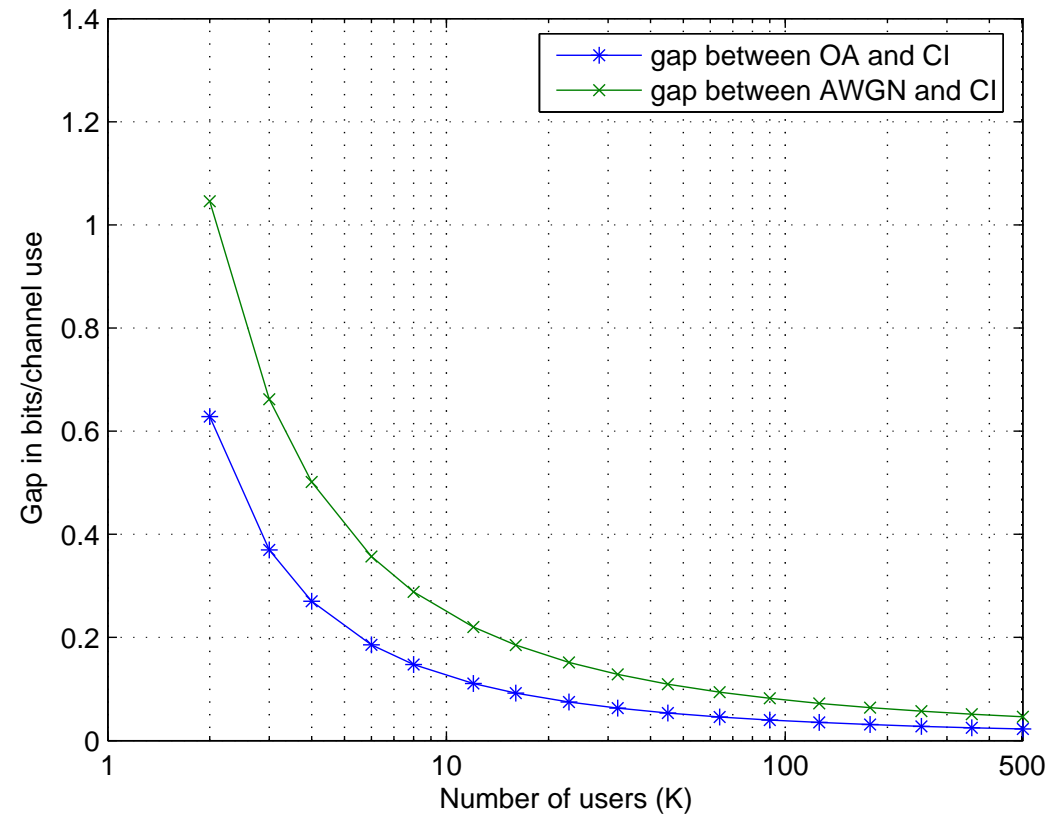

Fig. 8. $\lim _{S \rightarrow \infty}\left(C_{\mathrm{OA}}-C_{\mathrm{CI}}\right)$ and $\lim _{S \rightarrow \infty}\left(C_{\mathrm{AWGN}}-C_{\mathrm{CI}}\right)$ with single transmit and receive antennas and multi-user diversity 